Aus dem Institut für Diagnostische und Interventionelle Neuroradiologie Prof. Dr. med. Christian Riedel der Medizinischen Fakultät der Universität Göttingen

\title{
Vergleich der neuen Generation der Flachdetektor-CT (FDCT) mit der Multidetektor-CT (MDCT) bezüglich der Detektion von ischämischen und hämorrhagischen intrakraniellen Läsionen
}

\author{
INAUGURAL-DISSERTATION \\ zur Erlangung des Doktorgrades \\ der Medizinischen Fakultät der \\ Georg-August-Universität zu Göttingen
}

vorgelegt von

Johanna Rosemarie Leyhe

aus

Göttingen

Göttingen 2020 
Dekan: Prof. Dr. med. W. Brück

\section{Betreuungsausschuss}

Betreuer/in:

Prof. Dr. med. M.N. Psychogios

Ko-Betreuer/in:

Prof. Dr. med. J. Liman

\section{Prüfungskommission}

Referent/in:

Prof. Dr. med. M.N. Psychogios

Ko-Referent/in:

Prof. Dr. med. V. Rohde

Drittreferent/in:

Prof. Dr. med. J. Liman

Datum der mündlichen Prüfung:

16.11.2021 
Hiermit erkläre ich, die Dissertation mit dem Titel "Vergleich der neuen Generation der Flachdetektor-CT (FDCT) mit der Multidetektor-CT (MDCT) bezüglich der Detektion von ischämischen und hämorrhagischen intrakraniellen Läsionen" eigenständig angefertigt und keine anderen als die von mir angegebenen Quellen und Hilfsmittel verwendet zu haben.

Göttingen, den 
Die Daten, auf denen die vorliegende Arbeit basiert, wurden teilweise publiziert:

Leyhe JR, Tsogkas I, Hesse AC, Behme D, Schregel K, Papageorgiou I, Liman J, Knauth M, Psychogios MN (2017): Latest generation of flat detector CT as a peri-interventional diagnostic tool: a comparative study with multidetector CT. J Neurointerventional Surg $\underline{9}$, 1253-1257

Die Ergebnisse dieser Dissertation wurde im Rahmen der 51. Jahrestagung der „Deutschen Gesellschaft für Neuroradiologie e.V.“ 2016 in Köln als Poster vorgestellt und mit einem Posterpreis ausgezeichnet(Abstracts: 51. Jahrestagung der Deutschen Gesellschaft für Neuroradiologie e.V. in Kooperation mit der 24. Jahrestagung der Österreichischen Gesellschaft für Neuroradiologie, 5.- 8. Oktober 2016, Gürzenich, Köln 2016).

Ebenfalls wurde die Ergebnisse dieser Dissertation im Rahmen der „International Stroke Conference ISC)“ 2017, 22.-24.02.2018 in Houston, Texas als Postervortrag vorgestellt. 


\section{Inhaltsverzeichnis}

Abbildungsverzeichnis ........................................................................................ I

Tabellenverzeichnis ..................................................................................................... II

Abkürzungsverzeichnis .............................................................................. III

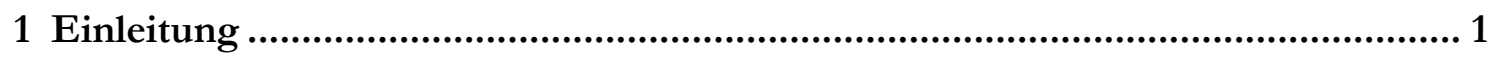

1.1 Neuroradiologische Schlaganfalldiagnostik und therapie ..............................................................

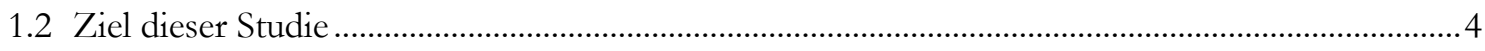

2 Zusammenfassende Darstellung der Methodik....................................................6

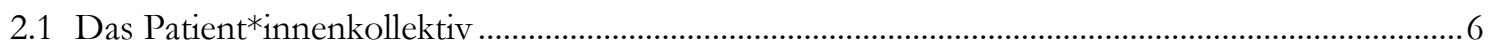

2.2 Technische Grundlagen der Computertomographie................................................................

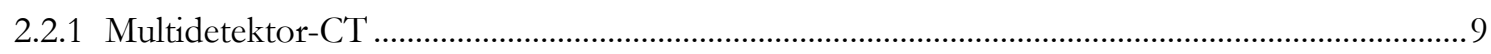

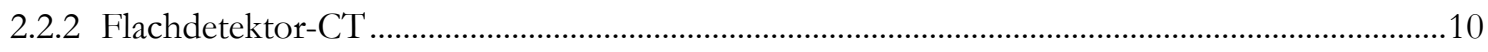

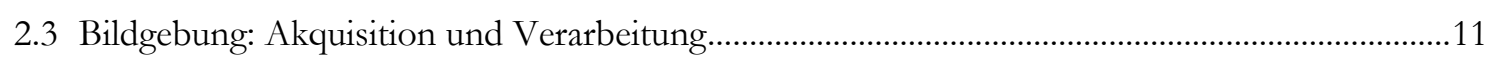

2.4 Analyse der Bildgebung und Statistik .......................................................................................

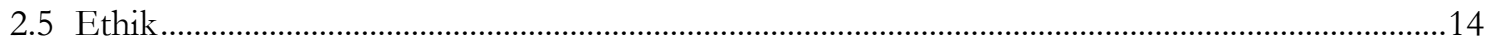

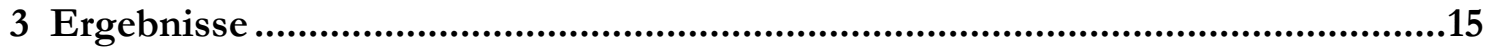

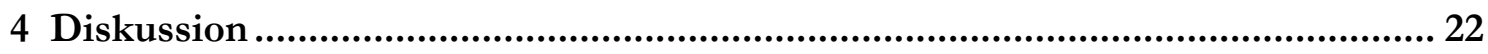

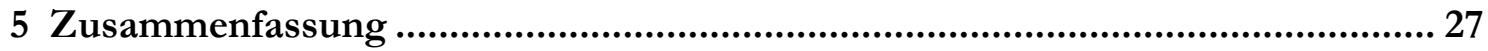

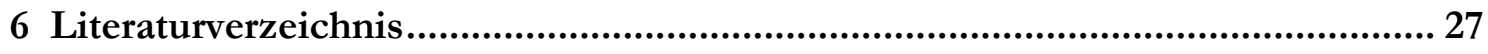

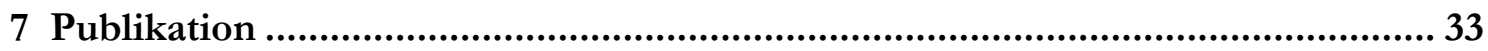

\section{Abbildungsverzeichnis}

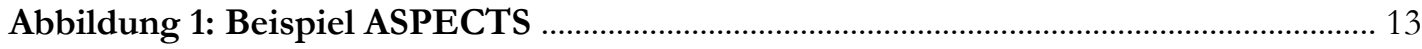

Abbildung 2: Bland Altman Plot der ASPECTS mittels FDCT und MDCT ................... 18 


\section{Tabellenverzeichnis}

Tabelle 1: Zusammensetzung des Patient*innenkollektivs 7

Tabelle 2: Ergebnisse der Auswertung der diagnostischen Beurteilbarkeit intrazerebraler Strukturen: Ventrikelsystem und Subarachnoidalräume 16 Tabelle 3: Ergebnisse der Auswertung der diagnostischen Beurteilbarkeit

intrazerebraler Strukturen: Grau-Weiß-Differenzierung 17

Tabelle 4: Blutungsdetektion ................................................................................................ 19

Tabelle 5: Einfluss der verschiedenen Artefakttypen .......................................................... 20 


\section{Abkürzungsverzeichnis}

$\begin{array}{ll}\text { ASPECTS } & \text { Alberta Stroke Program Early CT Score } \\ \text { AUC } & \text { area under the curve } \\ \text { CBF } & \text { Zerebraler Blutfluss } \\ \text { CBV } & \text { Zerebrales Blutvolumen } \\ \text { CT } & \text { Computertomographie } \\ \text { CTA } & \text { Computertomographie-Angiographie } \\ \text { CTP } & \text { Computertomographie-Perfusion } \\ \text { DGN } & \text { Deutsche Gesellschaft für Neurologie } \\ \text { FDCT } & \text { Flachdetektor-Computertomographie } \\ \text { HE } & \text { Houndsfield-Einheiten } \\ \text { ICB } & \text { Intrakranielle Blutung } \\ \text { IPB } & \text { Intraparenchymale Blutung } \\ \text { IVB } & \text { Intraventrikuläre Blutung } \\ \text { MDCT } & \text { Multidetektor-Computertomographie } \\ \text { mRS } & \text { modified Rankin Scale } \\ \text { MRT } & \text { Magnetresonanztomographie } \\ \text { rtPA } & \text { recombinant tissue plasminogen activator } \\ \text { SAB } & \text { Subarachnoidalblutung } \\ \text { SOP } & \text { standard operating procedure } \\ \text { VOxel } & \text { Volumetrisches Pixel }\end{array}$





\section{$1 \quad$ Einleitung}

\subsection{Neuroradiologische Schlaganfalldiagnostik und -therapie}

Ein Schlaganfall ist gemäß der Leitlinie Akuttherapie des ischämischen Schlaganfalls definiert als ein „ein akutes fokales neurologisches Defizit aufgrund einer umschriebenen Durchblutungsstörung des Gehirns [...]“ (DGN 2012). Wenn sich die klinischen Symptome hingegen innerhalb einer Stunde zurückbilden (Albers et al. 2002) beziehungsweise kein Korrelat in einer diffusionsgewichteten MRT-Bildgebung aufweisen (Easton et al. 2009), spricht man von einer transitorischen ischämischen Attacke (TIA). Ätiologisch liegt meist eine zerebrale Ischämie beispielsweise kardioembolischer, mikro- oder makroangiopathischer Genese vor. Seltener ist eine intrakranielle Blutung Ursache des Schlaganfalls, diese sind am häufigsten hypertensiv bedingt oder Folge einer Amyloidangiopathie (Hufschmidt et al. 2017). Als weltweit zweithäufigste Todesursache spielt der Schlaganfall eine zentrale Rolle im klinischen und insbesondere im neurologischen und neuroradiologischen Alltag und seine Behandlungsmethoden unterliegen einem ständigen Evaluations- und Forschungsprozess (Benjamin et al. 2017).

Für die Bildgebung hat sich die Computertomographie (CT) als Goldstandard-Methode etabliert, welche schnell und zuverlässig für die Diagnostik eingesetzt werden kann (Thomalla et al. 2009). Technisch gesehen stehen für diese Art der Bildgewinnung zwei verschiedene CTFormen zur Verfügung: einerseits die Flachdetektor-CT (FDCT), welche mit einem biplanen CBogen-System ausgestattet ist und Aufnahmen über einen mehr als $180^{\circ}$ großen Winkel erstellen kann, und die so genannte Multidetektor-CT (MDCT), welche Bilder mittels sequentieller Abtastung des zu untersuchenden Körperteils akquiriert. Der Einsatz der multiparametrischen Magnetresonanztomographie (MRT) kann prinzipiell ebenfalls für die Bildgebung im Rahmen der Schlaganfalldiagnostik eingesetzt werden. Die MRT ist der CT bezüglich der Darstellung früher Ischämien überlegen, steht allerdings nicht überall in räumlicher Nähe zur Verfügung und ist im Vergleich zur CT die zeitlich aufwendigere Untersuchung (DGN 2012).

Zum standardmäßigen Ablauf von Diagnostik und Behandlung zählt momentan eine initiale Bildgebung mittels Multidetektor Computertomographie, um die Genese des Schlaganfalls (hämorrhagisch oder ischämisch) zu ermitteln. Anschließend kommt es - sofern ein ischämischer Schlaganfall vorliegt und die Zeitfenster (siehe unten) und potentielle Kontraindikation beachtet wurden - zur Einleitung einer Thrombolyse-Therapie und/oder 
einer mechanischen Thrombektomie unter angiographischer Kontrolle mittels Flachdetektor Computertomographie.

Am selben Gerät wird abschließend der Ausschluss von Komplikationen wie beispielsweise einer Blutung o. ä. vorgenommen. Der Ablauf wird durch verschiedene Parameter definiert: Neben der Genese des Schlaganfalles spielt die Zeit zwischen Auftreten der Symptomatik und Therapiebeginn eine entscheidende Rolle. Als Zeitfenster für eine medikamentöse Thrombolysetherapie sind viereinhalb Stunden (Emberson et al. 2014) als europäischer Goldstandard festgelegt. Eine Erweiterung des Zeitfensters kann aktuell in Ausnahmefällen auf dem Boden der sogenannten „Wake Up Studie“ (Thomalla et al. 2018) ) unter Verwendung erweiterter Bilgebungsaramter. Mismatch-Bildgebung, Kollateraldarstellung im Rahmen eines „individuelle[r]n Heilversuch[s] zur Anwendung kommen.“(DGN 2015).

Für eine mechanische Thrombektomie liegt das Zeitfenster bei sechs Stunden (Easton et al. 2009; Goyal et al. 2016). Im Anschluss daran kann durch eine erweiterte Bildgebung (zum Beispiel durch die CT-Perfusion) der Effekt auf das klinische Outcome der Patientinnen und Patienten und damit die Entscheidung für oder gegen die mechanische Thrombektomie individuell evaluiert werden (DGN 2015; Psychogios et al. 2017a). Insgesamt unterliegen diese Kriterien bezüglich der mechanischen Thrombektomie einer aktuellen Evaluation, welche bislang noch keinen Einzug in die Leitlinien der deutschen Fachgesellschaften gefunden hat. So zeigt bezüglich des Zeitfensters für eine mechanische Thrombektomie die im Januar 2018 veröffentlichte DAWN-Studie (,DWI or CTP Assessment with Clinical Mismatch in the Triage of Wake-Up and Late Presenting Strokes Undergoing Neurointervention with Trevo"), dass Patientinnen und Patienten bezüglich ihres klinischen Outcomes auch in einem Zeitfenster von 6 bis 24 Stunden von einer mechanischen Thrombektomie profitieren können (Nogueira et al. 2017).

Dieser Ablauf ist insgesamt in den letzten Jahren mehrfach diskutiert und evaluiert worden: 2015 konnte durch mehrere multizentrische, randomisierte Studien der Therapieverlauf entscheidend verändert werden. Verglichen wurde zunächst die Methodik zur Intervention. Entgegen der damaligen Studienlage konnte erstmalig eine Überlegenheit der mechanischen Thrombektomie und Thrombolysetherapie gegenüber einer alleinigen Thrombolysetherapie nachgewiesen werden (Berkhemer et al. 2015; Campbell et al. 2015; Goyal et al. 2015).

Ebenfalls wurde die Wichtigkeit einer schnellen und effektiven Behandlung für eine frühe Reperfusion nach Symptombeginn für das Outcome der Patientinnen und Patienten im Sinne des time-is-brain-Konzeptes betont (Smith et al. 2005; Broderick et al. 2013; Kurz et al. 2013). Dieses Konzept beruht vor allem auf der den Infarktkern umgebenden ischämischen Penumbra, welche als so genannte tissue-at-risk-Region vor dem Gewebeuntergang bewahrt werden kann, 
vorausgesetzt es kommt zu einer frühen und erfolgreichen Reperfusion des betroffenen Areales. Durch den Schutz der gefährdeten Bereiche können das Auftreten neurologischer Defizite, die Mortalität sowie Morbidität gesenkt werden, wodurch das klinische Outcome deutlich verbessert werden kann (Gomez et al. 2002; Leary et al. 2003).

Ebenfalls hat sich die Spezialisierung der in die Schlaganfalldiagnostik und -therapie involvierten Mitarbeiterinnen und Mitarbeiter zu einem wichtigen Einflussfaktor auf das klinische Outcome entwickelt und unterliegt einer ständigen Qualitätskontrolle und -entwicklung (DGN 2012; Maier et al. 2013). So wird für Schlaganfall-Patientinnen und Patienten eine interdisziplinäre Behandlung auf einer so genannten Stroke-Unit empfohlen: Dort kann eine klinische Akutversorgung sowie Einleitung einer sekundärprophylaktischen Therapie und frührehabilitativer Maßnahmen gemeinsam mit Pflegekräften, Physiotherapeut*innen, Ergotherapeut*innen, Logopäd*innen und Sozialarbeiter*innen gewährleistet werden. Dies soll unter zertifizierten Qualitätsstandards erfolgen, um das klinische Outcome der Patientinnen und Patienten hinsichtlich Lebensqualität und Wiedereingliederung in den Alltag durch Reduktion von beispielsweise persistierenden funktionellen Defiziten $\mathrm{zu}$ verbessern. Auch soll eine engmaschige Überwachung des neurologischen Status sowie der allgemeinen Vitalparameter zur Reduktion dieser Komplikationen und zur Senkung der Mortalitätsrate beitragen (DGN 2012). In einer 2015 veröffentlichten Ergänzung zur Leitlinie „Akuttherapie des ischämischen Schlaganfalls“ (DGN 2015) werden ebenfalls direkte Qualitätsstandards für das neuroradiologische Arbeiten gestellt: So soll die mechanische Thrombektomie „Zentren mit entsprechender Erfahrung vorbehalten (... “ und „nur von darin ausgebildeten Interventionalisten (z. B. DGNR Zertifizierung Modul E)“ (DGN 2015) durchgeführt werden. Der zugrunde liegende Kontroll- und Evaluationsprozess soll mittels von „zur Qualitätssicherung prospektiv [erfassten] Leistungszahlen (z. B. Door-to-imaging-Zeit, Door-togroin-Zeit, Rekanalisationsrate etc.)“(DGN 2015) sichergestellt werden.

Auch in Hinblick auf die technischen Grundlagen wurden Schlaganfalldiagnostik und -therapie weiterentwickelt. Seit 2005 sind Flachdetektoren in der Lage, sogenannte cone beam volume images zu produzieren (Heran et al. 2006). Bei dieser Form der Bildgebung handelt es sich um ein dosiseffizientes Verfahren mit reduzierter Anfälligkeit für Artefakte und verbesserter Kontrastauflösung, welche eine postinterventionelle Komplikationskontrolle ohne Rücktransport zur MDCT möglich machte (Heran et al. 2006). Auch während einer Intervention kann eine solche Kontrolle notwendig werden, sollte sich beispielsweise die klinische Situation des Patienten/ der Patientin durch das ärztliche Eingreifen bedingt oder aufgrund der Schwere des vorhandenen Krankheitsbildes verschlechtern. 
Limitiert wurde ein breiterer Einsatz vor allem durch die mangelhafte Parenchymdarstellung insbesondere im infratentoriellen Bereich, der Artefakt-Anfälligkeit, sowie der begrenzten Möglichkeiten zur Detektion von intrakraniellen Ischämien und zum Teil auch durch Defizite im Bereich der Blutungsdetektion (Kau et al. 2014; Doerfler et al. 2015).

Durch die neuste Generation von Flachdetektor-CT kann nun die Möglichkeit geboten werden, zuverlässiger Blutungen und Ischämiezeichen zu erkennen und darauf aufbauend eine diagnostische Entscheidung treffen zu können. Als technische Grundlage hierfür fungiert ein Hochkontrast-Flachdetektor, der vor allem die Darstellung von Weichgewebe verbessert und eine Erweiterung der Rekonstruktionsalgorithmen bietet, welche den Einfluss der Artefakte auf die Bildqualität reduziert.

Der Begriff der Ischämie-Frühzeichen umfasst verschiedene Bildgebungsparameter, welche computertomographisch einen ersten wichtigen Hinweis für das Vorliegen eines ischämischen Schlaganfalls liefern können. Dazu zählen das hyperdense Mediazeichen, eine Dichteminderung im Linsenkern und das Verstreichen der Gyri und Sulci. Das Vorhandensein eines oder mehrerer dieser Zeichen erhärtet die klinische Diagnose eines Schlaganfalls und bedarf ebenfalls einer ausreichenden Bildqualität insbesondere hinsichtlich der auch in dieser Studie untersuchten Grau-Weiß-Differenzierung. Eine suffiziente Qualität der Grau-WeißDifferenzierung ist auch zum Erheben des ASPECTS notwendig. Dieser Score umfasst eine bildgebende Auswertung des Versorgungsterritoriums der Arteria cerebri media (ACM), welches quantitativ mit zehn (kein Hinweis auf ischämische Läsionen) bis eins (ischämische Läsionen im gesamten Stromgebiet der ACM) ausgewertet wird, wobei für jedes betroffene Gebiet ein Punkt vom Ausgangswert der zehn Punkte abgezogen wird. Die einzelnen Gebiete werden unter 2.4 mittels einer Abbildung genauer aufgeführt.

Eine mögliche Perspektive der erweiterten Einsatzmöglichkeiten der Flachdetektor-CT könnte durch die verbesserten diagnostischen Möglichkeiten der Einsatz als bildgebendes Verfahren im so genannten one-stop-management sein (Yang et al. 2015; Psychogios M-N et al. 2017b). Dieses Konzept sieht einen direkten Transport des/der Patient*in in den Angiographie-Raum zur FDCT vor, wodurch Diagnostik und Therapie nur noch an einem CT-Gerät stattfinden kann. Dadurch wird einerseits die Zeit bis zur Reperfusion reduziert, zum anderen werden durch die Umlagerung bedingte Komplikationen reduziert (Doerfler et al. 2015).

\subsection{Ziel dieser Studie}

Grundsätzliches Ziel der Studie ist der multifaktorielle Vergleich von Flachdetektor-CT und Multidetektor-CT. Beide Geräte sind momentan Teil des diagnostischen Prozesses im Rahmen 
einer Schlaganfallbehandlung: Zunächst wird der/die Patient*in nach der klinischen Verdachtsdiagnose zur Multidetektor-CT gebracht und bekommt die erste Bildgebung mit dem primären Ziel, eine Blutung auszuschließen und/oder eine Ischämie feststellen zu können. Fällt der Entschluss zur Intervention, wird der/die Patient*in in den Angiographie-Raum transportiert und dort unter Kontrolle mittels Flachdetektor-CT behandelt. Abschließend erhält der/die Patient*in hier eine Bilderserie zum Ausschluss einer Komplikation, wie beispielsweise einer Blutung.

Um die Bildqualität der beiden Verfahren zu evaluieren wurden folgende Faktoren von drei unterschiedlich erfahrenen Auswerter*innen analysiert: zunächst die Darstellung des Ventrikelsystems sowohl supra- als auch infratentoriell und des Parenchyms im Bereich der Basalganglien, der Insel, der Zentralregion und des Cerebellums. Darüber hinaus wird für jeden Patienten und jede Patientin ein ASPECTS (siehe Abbildung 1) erhoben. Ebenfalls wird die Detektion von Blutungen (Subarachnoidalblutung, intraventrikuläre Blutung, intraparenchymale Blutung) evaluiert und die Anzahl der Bildschichten vergleichen, welche die Blutung zeigen. Bei intraparenchymalen Blutungen wird noch zusätzlich der Blutungsdurchmesser an der Stelle der größten Ausdehnung der Blutung gemessen und verglichen. Einen weiteren Punkt bildet der Einfluss der verschiedenen Artefakttypen. Verglichen werden Metall-, Bewegungs-, Ring-, Streifen- und Einbrandartefakte. Abschließend wird der subjektive Eindruck der Auswertenden festgehalten, inwieweit mit den vorliegenden Aufnahmen ein sicheres Erkennen einer Blutung oder einer Ischämie möglich sei.

Bei den drei Auswerter*innen handelt es sich um einen erfahrenen Facharzt mit der Zusatzbezeichnung für Neuroradiologie ( $>5$ Jahre Berufserfahrung), um einen Assistenzarzt ( $<5$ Jahre Berufserfahrung) und um eine Medizinstudentin und Doktorandin der Arbeitsgruppe Klinische Schlaganfallforschung. 


\section{Zusammenfassende Darstellung der Methodik}

\subsection{Das Patient*innenkollektiv}

Grundlage dieser Studie bildete die retrospektive Analyse aller angiographischen Behandlungen im Institut für Diagnostische und interventionelle Neuroradiologie der Universitätsmedizin Göttingen seit Installation einer neuen Generation der Flachdetektor-CT im September 2014 bis einschließlich April 2016.

Als Einschlusskriterium wurde festgelegt, dass alle Patient*innen neben der periinterventionellen FDCT-Bildgebung auch über eine postinterventionelle MDCTBildgebung verfügen mussten. Das Patient*innenkollektiv umfasste somit insgesamt 105 Patient*innen. Im darauffolgenden Schritt wurden drei Personen aus der Studie ausgeschlossen, da die Bildgebung aufgrund zu starker Bewegungsartefakte nicht auswertbar war. Bezüglich der Geschlechterverteilung setzte sich das Kollektiv aus 50 Frauen und 52 Männern zusammen, welche eine Altersspanne von 22 Jahre bis 90 Jahre bei einem medianen Alter von 63 Jahren aufwiesen.

Die Mehrheit (48 Personen) der Patientinnen und Patienten wurde mittels einer Thrombektomie behandelt, weitere 24 Personen erhielten ein Coiling, 12 Personen ein Stenting und ein Patient wurde mit einem neurochirurgischen Clip behandelt. 17 Patientinnen und Patienten erhielten eine digitale Subtraktionsangiographie (DSA). Tabelle 1 gibt einen Überblick über die Zusammensetzung des Patient*innenkolletivs. 
Tabelle 1: Zusammensetzung des Patient*innenkollektivs

\begin{tabular}{|l|l|}
\hline Merkmal & $\begin{array}{l}\text { Patient*innen } \\
\text { (n= 102) }\end{array}$ \\
\hline Alter: Median (Bereich) [Jahre] & $63(22-90)$ \\
\hline Geschlecht männlich [Anzahl (\%)] & $52(50,9)$ \\
\hline Diagnostische/therapeutische Behandlung [Anzahl (\%)] \\
\hline Thrombektomie & $48(47,1)$ \\
\hline Coil & $24(23,5)$ \\
\hline DSA & $17(16,6)$ \\
\hline Stent & $12(11,7)$ \\
\hline Clip & $1(0,98)$ \\
\hline
\end{tabular}

\subsection{Technische Grundlagen der Computertomographie}

Die Computertomographie ist ein Röntgenverfahren mit dessen Hilfe sich Schnittbilder erzeugen lassen, welche sich im Gegensatz zu den Projektionsdaten konventioneller Röntgengeräte überlagerungsfrei darstellen lassen. Hierfür wird das zu untersuchende Objekt/ Patient*in je nach Gerätetyp röhren- oder spiralförmig entlang der z-Achse abgetastet und die Abschwächung der Röntgenstrahlung von einem Detektor erfasst, wobei die Strahlungsbreite durch eine Blendung registriert wird (Kramme 2017). Die registrierte Abschwächung kann anschließend in ein elektrisches Signal umgewandelt und zu Rohdaten weiterverarbeitet werden. Die Besonderheit bei der spiralförmigen Abtastung durch Rotation der Röntgenröhre und den kontinuierlichen Tischvorschub ist die Möglichkeit verschiedener Rekonstruktionen aus einem Volumendatensatz, wie zum Beispiel dreidimensionale Darstellungen. Für einen Volumendatensatz wird für jeden durchleuchteten Punkt die individuelle Abschwächung errechnet (Grillenberger und Fritsch 2012).

Die Röntgenstrahlung wird von den unterschiedlichen Gewebearten verschieden stark abgeschwächt und die sich daraus ergebenen Schwächungswerte von dem Detektor registriert. Aus diesen Werten wird für jedes Pixel ein Röntgenabsorptionskoeffizient ermittelt, der in so genannte Graustufen übersetzt und dann in Hounsfield-Einheiten (HE) angegeben wird, um eine geräteunabhängige Skalierung vornehmen zu können. Für diese Skala wurde für Wasser der Wert 0 HE definiert, insgesamt reicht sie von -1000 HE (Luft) bis + 3000 HE (Kramme 2017). Um die Wahrnehmung der einzelnen Graustufen für das menschliche Auge zu verbessern, 
können für unterschiedliche Fragestellungen verschiedene Darstellungsformen gewählt werden, so genannte Fenster (z. B. Knochenfenster oder Weichteilfenster). Diese Fenster fassen bestimmte Hounsfieldwerte zusammen, um die für die Fragestellung relevanten Bereiche gut darzustellen.

Betrug die Akquisitionszeit früherer Generationen noch um die 300 Sekunden pro Schicht, beträgt diese mit modernen Mehrzeilen-CT weniger als eine Sekunde pro Schicht (Kramme 2017). Als weitere Bildgebungsverfahren spielen die Computertomographie-Perfusion (CTP) und die Computertomographie-Angiographie (CTA) eine wichtige Rolle in der neuroradiologischen Bildgebung. Die CT-Perfusion stellt mittels verschiedener Parameter die zerebrale Durchblutung dar, gemessen werden der zerebrale Blutfluss (CBF), das zerebrale Blutvolumen (CBV), die mittlere Transitzeit des Kontrastmittel-Bolus (MTT) und die time-topeak (TTP), was der Zeit von der Bolusgabe bis zur maximalen Kontrastmittelanreicherung im Extrazellularraum entspricht (Hosten und Liebig 2000). Im Rahmen der Schlaganfalldiagnostik kann mittels der CTP das Zeitfenster von sechs Stunden gegebenenfalls erweitert werden (DGN 2015; Psychogios et al. 2017a): Für die Darstellung der Penumbra, das Gewebe, welches sich um den Infarktkern befindet und potentiell noch von einer Reperfusion profitieren kann (tissueat-risk), kann ein sogenanntes Missverhältnis (mismatch) errechnet werden. Hierfür muss das $\mathrm{CBV}$, welches dem Infarktkern entspricht, von dem CBF abgezogen werden, welcher das gesamte betroffene Areal anzeigt.

Bei der CT-Angiographie können nach intravenöser Kontrastmittel-Applikation die Schnittbilder zu einer dreidimensionalen Gefäßdarstellung verrechnet werden, welche sich frei drehen lässt und somit verschiedene Pathologien der Gefäße detektiert werden können (Thomalla et al. 2009). Auch können mittels der CTA mögliche Kollateralgefäße dargestellt werden, welche beispielweise bei einem Gefäßverschluss weiterhin Teile der betroffenen Region perfundieren und somit die Prognose für das klinische Outcome positiv beeinflussen können (Liebeskind et al.

2014; Schregel et al. 2018). 


\subsubsection{Multidetektor-CT}

Bei der Multidetektor-CT handelt es sich um eine Technologie, bei welcher mehrere Detektoren um die auf dem Tisch liegenden Patient*in rotieren. Im Vergleich zu früheren Generationen von CT-Geräten können durch den Einsatz mehrerer Detektoren größere Untersuchungsvolumina in einer kürzeren Zeit akquiriert werden (Grillenberger und Fritsch 2012). Durch die Verkürzung der Untersuchungszeit verringert sich das Auftreten von Bildartefakten. Dadurch können Bilder mit einer geringen Schichtdicke erzeugt werden, was eine Weiterverarbeitung ohne Verlust einer guten räumlichen Auflösung ermöglicht (Grillenberger und Fritsch 2012). Die Entwicklung vollzog sich über mehrere Generationen von CT-Geräten.

Die Technik der CT beruht auf einem bzw. je nach Anzahl der Detektoren mehreren Fächerstrahlen, welche durch Kollimation zur gewünschten Größe gebracht werden. Durch die parallele Schaltung mehrerer Detektoren kann deren Breite variiert werden und somit die Ortsauflösung anhand der minimalen Schichtdicke bestimmt werden (Kramme 2017). Bezüglich der Bildrekonstruktion wird zwischen Rohdaten und Bilddaten unterschieden. Aus den während der Abtastung gewonnenen Daten werden durch ein Weiterverarbeitungsprogramm die Rohdaten erzeugt, welche anschließend vom Detektor in Bilddaten umgewandelt werden. Dies geschieht unter der Berücksichtigung der verschiedenen Rekonstruktionsparameter wie der z-Filterung, des Faltungskerns und der Bildmatrix (Grillenberger und Fritsch 2012). Über diese Rekonstruktionsparameter werden verschiedene Bildparameter wie Ortsauflösung, Kanten oder Rauschen festgelegt, welche an die darzustellende Struktur (z. B. abhängig von Hoch- und Niedrigkontrastbildgebung) oder auch verschiedenen Indikationsstellungen angepasst werden können. 


\subsubsection{Flachdetektor-CT}

Die so genannte Flachdetektor Computertomographie wird im Wesentlichen als C-BogenSystem im klinischen Alltag eingesetzt. Zunächst basierten diese Systeme auf dem Einsatz von Bildverstärkern, welche jedoch aufgrund ihrer limitierten technischen Leistungsfähigkeit hauptsächlich im Bereich der 3D-Rotationsangiographie eingesetzt werden konnten. Um dieses Anwendungsspektrum in Hinblick auf das der konventionellen CT zu erweitern, wurden die Bildverstärkersysteme durch die so genannten FDCT-C-Bogen-Systeme weitestgehend ersetzt. Diese Systeme generieren mit einem Winkel von über $180^{\circ}$ innerhalb weniger Sekunden $500-$ 600 Einzelaufnahmen (Psychogios et al. 2010). In Bezug auf einen möglichen Einsatz im Bereich der Schlaganfalldiagnostik konnte durch diese Systemumstellung beispielsweise eine Verbesserung in der Weichgewebskontrastdarstellung erreicht werden, welche für die Detektion von intrakraniellen Blutungen essentiell ist (Kyriakou et al. 2009). Die Detektoren konvertieren Röntgenstrahlen in ein elektrisches Signal. Hierfür werden die Röntgenstrahlen zunächst durch einen Szintillator wie Caesium-Iodid in Photonen umgewandelt. Photodioden verarbeiten dieses sichtbare Licht in ein elektrisches Signal, aus welchem durch eine digitale Weiterverarbeitung das eigentliche Röntgenbild entsteht (Kyriakou et al. 2009).

Dieser Prozess kann durch verschiedene Parameter beeinflusst werden: Beispielsweise kann durch die Auswahl des Szintillatormaterials die Absorption der Röntgenstrahlung beeinflusst werden, was sich wiederrum auf die Ortsauflösung auswirkt. Durch die Verwendung dünner Materialien werden die Ausbreitungsmöglichkeiten im Material selbst reduziert und somit die Ortsauflösung verbessert, bei dickeren Materialien kommt es zu einem umgekehrten Effekt (Kyriakou et al. 2009). Ein gängiges Material ist das oben aufgeführte Caesium-Iodid. Um dem Problem der, mit dieser Form der Akquisition verbundenen, zu geringen Bildrate zu entgehen, wird auf das Prinzip des Binnings, einer Kombination mehrerer Pixel, zurückgegriffen, wodurch eine veränderte Matrix und eine höhere Bildrate pro Sekunde entsteht (Kyriakou et al. 2009).

Die für die Weiterverarbeitung benötigten Volumendatensätze lassen sich bei der FDCT durch eine Röhrenspannung von ca. 109 kV gewinnen. Hierbei kann durch die Belichtungsautomatik sowohl der Röntgenstrom als auch die Röntgenspannung reguliert werden, wobei für die CTBildgebung eine konstante Spannung von Vorteil ist, um der Entstehung weiterer Bildartefakte vorzubeugen (Kyriakou et al. 2009). Anschließend kann eine Weiterverarbeitung beispielsweise zu einem 3D-Datensatz erfolgen.

Im Rahmen dieser Studie kam die Flachdetektor-CT gemäß ihrer regulären klinischen Anwendung zum Einsatz. Für die Schlaganfalldiagnostik und -therapie wurde sie primär als 
Angiographie-CT und somit als bildgebende Komponente während eines interventionellen Eingriffs genutzt. Für die postinterventionelle Kontrolle zum Ausschluss von Komplikationen, wie beispielsweise einer intrakraniellen Blutung, wurde ebenfalls die FDCT wie in der allgemeinen klinischen Routine verwendet.

\subsection{Bildgebung: Akquisition und Verarbeitung}

Für Bildakquisition und endovaskuläre Intervention wurde eine Flachdetektor CT (Artis QBiplane, Siemens Healthcare GmbH, Erlangen, Germany) und eine Multidetektor CT (Somatom Definition AS+, Siemens Healthcare GmbH, Erlangen, Germany) verwendet. Als Protokoll wurden 20 Sekunden für die Akquisition gefahren, wie auch in der klinischen Routine des Instituts. Das standardmäßige Protokoll beinhaltete weiterhin die Anfertigung von 538 Projektionen, welche durch eine 20 Sekunden dauernde Rotation des C-Bogens bei einem Winkel von $220^{\circ}$ möglich sind (Kalender und Kyriakou 2007). Die Röntgendosis der FDCT lag hierfür bei 35 mGy. Insgesamt kann durch den 30 x $40 \mathrm{~cm}$ großen Flachdetektor eine Aufnahmehöhe von $16 \mathrm{~cm}$ bei einer Aufnahmebreite von $22 \mathrm{~cm}$ erzeugt werden (Psychogios et al. 2010).

Für die Bildrekonstruktion wurde eine syngoXWP VC10N-Workstation (Siemens Healthcare GmbH, Erlangen, Germany) mit einer 3D InSpace Software verwendet und die Bilder für die retrospektive Analyse rekonstruiert. Dafür wurde ein weicher Faltungskern für eine optimale Kontrastauflösung gewählt, was dem Standard der Parenchymdarstellung anhand dieses Gerätetyps entspricht. Um die CT-ähnlichen Bilder an sich zu produzieren, wurde im nächsten Schritt ein Volumendatensatz mit einer Bildmatrix von 512 x 512 isotropischen Pixeln rekonstruiert. Eine abschließende Ausrichtung beider Bilddatensätze erfolgte anhand der orbitomeatalen Linie mit einer Bilddicke von $5 \mathrm{~mm}$ und einem Bildabstand von $3 \mathrm{~mm}$ sowie mit insgesamt 40 bis 50 Einzelschichten. Bezüglich des zeitlichen Ablaufes wurden die Patientenbilder zeitnah nach deren Therapie rekonstruiert und anonymisiert auf der Workstation gespeichert.

\subsection{Analyse der Bildgebung und Statistik}

Die Speicherung und Analyse der erhobenen Bilddaten erfolgte retrospektiv an einer syngoXWP VC10N-Workstation (Siemens Healthcare GmbH, Erlangen, Germany. Die patientenbezogenen Daten wurden nach der Rekonstruktion bezüglich der Angaben zum 
Namen, Geburtstag und Art der Untersuchung anonymisiert und anschließend unabhängig von jedem Auswerter evaluiert. Dafür wurden die Bilder zufällig in zwei Gruppen eingeteilt, welche aus FDCT- und MDCT-Scans bestanden. Die Evaluation durch die Auswertenden fand mit einem zeitlichen Abstand von mindestens dreißig Tagen statt, um einem möglichen Bias vorzubeugen.

Bei der Gruppe der Auswerter handelte es sich bei NRAD 1 um einen sehr erfahrenen Neuroradiologen ( $>5$ Jahre Berufserfahrung), bei NRAD 2 um einen durchschnittlich erfahrenen Neuroradiologen ( $<5$ Jahre Berufserfahrung) und bei NRAD 3 um eine MedizinDoktorandin mit geringer neuroradiologischer Erfahrung. Alle Auswerter*innen nutzten den gleichen Fragebogen und konnten für die Bildbetrachtung ihre Fensterung frei wählen.

Der Fragebogen gliederte sich im Wesentlichen in vier verschiedene Themenkomplexe: die Analyse des Liquorsystems, Beurteilung der Parenchymdiagnostik und der potentiellen Ischämiedetektion, Analyse der potentiellen Blutungsdetektion und die Bewertung potentieller verschiedener Artefakttypen.

Für die Analyse des Liquorsystems wurde die Darstellung des Ventrikelsystems und des Subarachnoidalraums im supra- und im infratentoriellen Bereich ausgewertet. Anschließend wurde der Punkt der Parenchymdarstellung evaluiert, indem für die Schlaganfalldiagnostik relevante intrakranielle Regionen wie die Basalganglien, Insula, Zentralregion und Cerebellum einzeln betrachtet wurden. Die Analyse der beiden Themenkomplexe erfolgte anhand einer dreistufigen Bewertungsskala mit den Möglichkeiten „gut beurteilbar“ (1), „ausreichend diagnostisch beurteilbar“ (2) und „nicht beurteilbar“ (3).

Zusätzlich wurde von den Auswertern der ASPECTS für jeden Patienten erhoben. Beim ASPECTS handelt es sich um ein zehn Punkte umfassendes, quantitativ-topographisches Bewertungssystem. Hierfür wird das Territorium der Arteria cerebri media in zehn verschiedene Regionen unterteilt, welche auf Ischämiezeichen untersucht werden. Ein ASPECTS von 10 Punkten sagt somit aus, dass kein Ischämiezeichen vorliegt(Liebeskind et al. 2014; Schregel et al. 2018). 

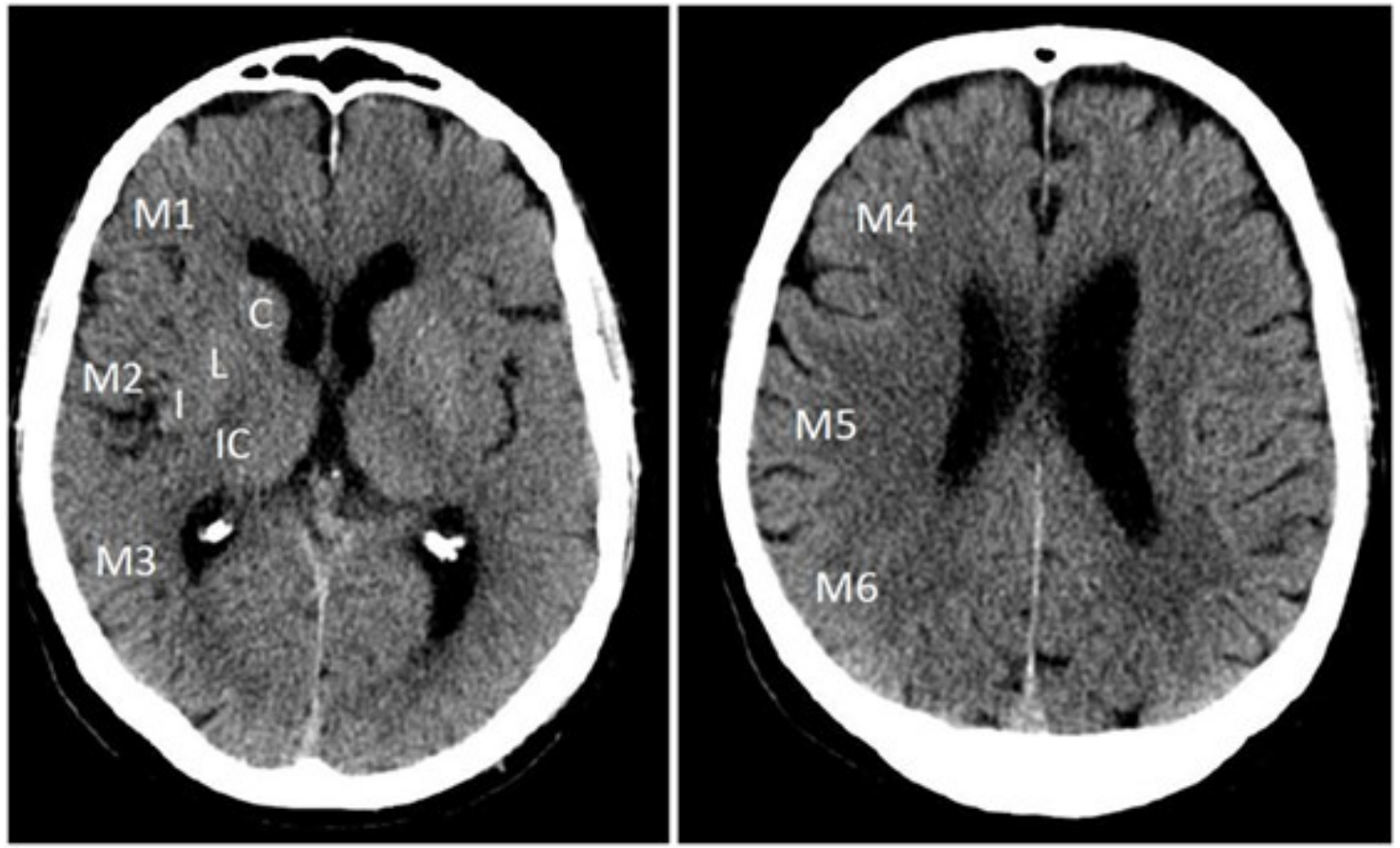

\section{Abbildung 1: Beispiel ASPECTS}

Die Abbildung zeigt ein beispielhaften ASPECTS eines CT-Bildes: M1 bezeichnet den vorderen Media- Kortex, M2 den mittleren Kortex seitlich der Inselregion und M3 den hinteren Kortex. M4-M5 entsprechen den vorderen, seitlichen und hinteren Mediastromgebieten unmittelbar über M1-M3. I steht für den Inselkortex, IC für die Capsula interna, L bezeichnet den Linsenkern und C das Caput nucleus caudati (Schröder und Thomalla 2017)(CC BY 4.0).

Einen umfangreichen Bereich bildete die Blutungsanalyse: zunächst wurde, sofern eine Blutung vorhanden war, diese klassifiziert (Subarachnoidalblutung, Intraventrikuläre Blutung, Intraparenchymale Blutung) und danach die Anzahl der orbitomeatalen Schichten gezählt, welche die Blutung zeigten. Für die intraparenchymalen Blutungen wurde zusätzlich der Blutungsdurchmesser bestimmt. Die ausgewerteten Blutungen wurden anhand der Anzahl an Schichten, welche die Blutung zeigten, in drei Gruppen unterteilt: 0-25. Perzentile (,klein“), 2575. Perzentile (,mittelgroß“) und 75-100. Perzentile (,groß`).

Anschließend wurde der Einfluss verschiedener Artefakttypen (siehe Tabelle 5) beurteilt, wobei eine Unterteilung, wie bereits bei 1.2 „Ziel dieser Studie“ erläutert, vorgenommen wurde. Die Auswerter verwendeten hierfür eine fünfstufige Skala: „,nicht vorhanden“ (0), „kaum sichtbar“ (1), „sichtbar“ (2), „,beeinträchtigen Auswertung wesentlich“ (3) und „machen Auswertung unmöglich“ (4). Ebenfalls wurde die subjektive Einschätzung bezüglich der Blutungsdetektion im supra- und im infratentoriellen Bereich in die Auswertung mit aufgenommen und mit einer fünfstufigen Skala abgebildet: „Nicht möglich“ (0), ,sehr gut“ (1), ,gut“ (2), „,mittelmäßig“ (3) und „kaum möglich“ (4). 
Abschließend gaben die Auswerter*innen an, ob ihrer Meinung nach mit der analysierten Aufnahme ein sicherer Ausschluss/ Erkennen einer Blutung oder eines großen Infarktes (ASPECTS 0-4) möglich gewesen sei.

Die statistische Analyse erfolgte mithilfe des Programmes MedCalc (MedCalc Software version 14, Ostend, Belgium). Als Signifikanzlevel wurde für alle Auswertungen a = 0,05 festgesetzt. Für die Auswertungen innerhalb einer Kategorie wurden Kontingenztabellen und der Wilcoxon-Test für gepaarte Stichproben verwendet. Der Vergleich kategorischer Variablen zwischen verschiedenen Kategorien wurde mittels Fischer-Tests berechnet. Für die Sensitivität und Spezifität der Detektion von Blutungen und Ischämien mit den FDCT-Aufnahmen wurden ROC-Kurvenanalysen durchgeführt, wobei die Aufnahmen der MDCT als Goldstandard verwendet wurden. Für die Berechnung des Durchmessers der intraparenchymalen Blutungen und der ASPECTS-Werte beider Modalitäten wurden Bland-Altman-Plots berechnet. Das Intraobserver agreement wurde mit dem Spearmanschen Randkorrelationskoeffizient ermittelt.

\section{$2.5 \quad$ Ethik}

Ein Votum der Ethikkommission der Universitätsmedizin Göttingen war nach Rücksprache mit deren Vorsitzenden Prof. Dr. Brockmöller nicht notwendig. Durch das retrospektive Studiendesign wurden alle Pateint*innen nach dem regulären klinischen Protokoll des Instituts für Diagnostische und Interventionelle Neuroradiologie behandelt, wodurch keine zusätzlichen Behandlungsmaßnahmen oder Bildgebung notwendig war. 


\section{Ergebnisse}

Insgesamt konnten 105 Patient*innen als geeignet ermittelt werden, von denen aufgrund massiver Bewegungsartefakte drei Personen ausgeschlossen wurden und somit 102 Patient*innen in diese Studie eingeschlossen wurden. Das Kollektiv umfasste 50 Frauen und 52 Männer mit einem medianen Alter von 63 Jahren. 48 Personen wurden mittels Thrombektomie behandelt, 24 Personen mittels Coiling, 17 Personen erhielten eine DSA, 12 Personen wurden mit einem Stent behandelt und eine Person erhielt einen neurochirurgischen Clip (vgl. auch Kapitel 2.1).

Die Auswertung der Daten für die Darstellung des supratentoriellen Ventrikelsystems durch die FDCT zeigte keinen Unterschied zu den für die MDCT-Aufnahmen erhobenen Daten (FDCT vs. MDCT: 102 (100\%) vs. 102 (100\%) ,gut beurteilbar“ (1); siehe Tabelle 2).

Ähnlich gleichwertige Ergebnisse zeigten die Auswertungen der supratentoriellen Subarachnoidalräume (FDCT vs. MDCT: 98 (96\%) vs. 99 (97\%) ,gut beurteilbar“ (1); p = 0,813; siehe Tabelle 2) und des infratentoriellen Ventrikelsystems (FDCT vs. MDCT: 92 (90\%) vs. 98 (96\%), gut beurteilbar“ (1); $\mathrm{p}=$ 0,106; siehe Tabelle 2).

Die Ergebnisse für den infratentoriellen Subarachnoidalraum zeigten einen Unterschied zwischen FDCT und MDCT (FDCT vs. MDCT: 75 (74\%) vs. 85 (83\%) ,gut beurteilbar“ (1); $\mathrm{p}=0,177$; siehe Tabelle 2).

Insgesamt zeigte sich kein statistisch signifikanter Unterschied zwischen beiden Modalitäten bezüglich der Auswertung des Ventrikelsystems und der Subarachnoidalräume.

Die Parenchymdarstellung mit der FDCT zeigte in dem Bereich der Basalganglien, des insulären Kortex und des zentralen Kortex vergleichbare Werte zwischen FDCT und MDCT. So betrug die Bewertung der Grau-Weiß-Differenzierung der Basalganglien 97 (95\%) „gut beurteilbar“ (1) für die FDCT und 99 (97\%), ,gut beurteilbar“ (1) für die MDCT ohne statistisch signifikanten Unterschied $(\mathrm{p}=0,563)$.

Beim insulären Kortex (FDCT vs. MDCT: 94 (92\%) vs. 96 (94\%) ,gut beurteilbar“ (1); p = 0,625; siehe Tabelle 3) und bei der Auswertung der Ergebnisse der Zentralregion (FDCT vs. MDCT: 98 (96\%) vs. 99 (97\%) „gut beurteilbar“ (1); p = 0,813; siehe Tabelle 3) zeigte sich ebenfalls kein signifikanter Unterschied.

Die Grau-Weiß-Differenzierung des Zerebellums (infratentorielle Region) auf den FDCTAufnahmen zeigte hingegen Einschränkungen mit einem statistisch signifikanten Unterschied 
zwischen beiden Modalitäten (FDCT vs. MDCT: 57 (56\%) vs. 88 (86\%) „gut beurteilbar“ (1); $\mathrm{p}<0,001 ;$ siehe Tabelle 3).

Tabelle 2: Ergebnisse der Auswertung der diagnostischen Beurteilbarkeit intrazerebraler Strukturen: Ventrikelsystem und Subarachnoidalräume

\begin{tabular}{|c|c|c|c|c|c|c|}
\hline & & $\begin{array}{l}\text { Gut } \\
\text { beurteilbar } \\
\text { (1) }\end{array}$ & $\begin{array}{l}\text { Ausreichend } \\
\text { diagnostisch } \\
\text { beurteilbar } \\
\text { (2) }\end{array}$ & $\begin{array}{l}\text { Nicht } \\
\text { beurteilbar } \\
\text { (3) }\end{array}$ & Gesamt & Wilcoxon \\
\hline Variable & & n $(\%)$ & n $(\%)$ & n (\%) & n (\%) & $\mathrm{p}$ \\
\hline $\begin{array}{l}\text { Supratentorielles } \\
\text { Ventrikelsystem }\end{array}$ & $\begin{array}{l}\text { FDCT } \\
\text { MDCT }\end{array}$ & $\begin{array}{l}102(100) \\
102(100)\end{array}$ & $\begin{array}{l}0(0) \\
0(0)\end{array}$ & $\begin{array}{l}0(0,0) \\
0(0,0)\end{array}$ & $\begin{array}{l}102(100) \\
102(100)\end{array}$ & - \\
\hline $\begin{array}{l}\text { Infratentorierelles } \\
\text { Ventrikelsystem }\end{array}$ & $\begin{array}{l}\text { FDCT } \\
\text { MDCT }\end{array}$ & $\begin{array}{l}92(90) \\
98(96)\end{array}$ & $\begin{array}{l}9(9) \\
3(3)\end{array}$ & $\begin{array}{l}1(1) \\
1(1)\end{array}$ & $\begin{array}{l}102(100) \\
102(100)\end{array}$ & 0,106 \\
\hline $\begin{array}{l}\text { Supratentorieller } \\
\text { Subarachnoidalraum }\end{array}$ & $\begin{array}{l}\text { FDCT } \\
\text { MDCT }\end{array}$ & $\begin{array}{l}98(96) \\
99(97)\end{array}$ & $\begin{array}{l}4(4) \\
3(3)\end{array}$ & $\begin{array}{l}0(0) \\
0(0)\end{array}$ & $\begin{array}{l}102(100) \\
102(100)\end{array}$ & 0,813 \\
\hline $\begin{array}{l}\text { Infratentorieller } \\
\text { Subarachnoidalraum }\end{array}$ & $\begin{array}{l}\text { FDCT } \\
\text { MDCT }\end{array}$ & $\begin{array}{l}75(74) \\
85(83)\end{array}$ & $\begin{array}{l}25(25) \\
14(14)\end{array}$ & $\begin{array}{l}2(2) \\
3(3)\end{array}$ & $\begin{array}{l}102(100) \\
102(100)\end{array}$ & 0,177 \\
\hline
\end{tabular}

FDCT: Flachdetektor-Computertomographie, MDCT: Multidetektor-Computertomographie. Die jeweiligen Prozentangaben für „n“ beziehen sich auf die Kollektivgröße von 102 Patient*innen. 
Tabelle 3: Ergebnisse der Auswertung der diagnostischen Beurteilbarkeit intrazerebraler Strukturen: Grau-Weiß-Differenzierung

\begin{tabular}{|c|c|c|c|c|c|c|}
\hline & & $\begin{array}{l}\text { Gut beur- } \\
\text { teilbar (1) }\end{array}$ & $\begin{array}{l}\text { Ausreichend } \\
\text { diagnostisch } \\
\text { beurteilbar } \\
\text { (2) }\end{array}$ & $\begin{array}{l}\text { Nicht } \\
\text { beurteilbar } \\
\text { (3) }\end{array}$ & Gesamt & Wilcoxon \\
\hline Variable & & n $(\%)$ & n $(\%)$ & n $(\%)$ & n $(\%)$ & $\mathrm{p}$ \\
\hline $\begin{array}{l}\text { Grau-Weiß- } \\
\text { Differenzierung } \\
\text { der Basalganglien }\end{array}$ & $\begin{array}{l}\text { FDCT } \\
\text { MDCT }\end{array}$ & $\begin{array}{l}97(95) \\
99(97)\end{array}$ & $\begin{array}{l}5(5) \\
3(3)\end{array}$ & $\begin{array}{l}0(0) \\
0(0)\end{array}$ & $\begin{array}{l}102(100) \\
102(100)\end{array}$ & 0,563 \\
\hline $\begin{array}{l}\text { Grau-Weiß- } \\
\text { Differenzierung } \\
\text { des insulären } \\
\text { Kortex }\end{array}$ & $\begin{array}{l}\text { FDCT } \\
\text { MDCT }\end{array}$ & $\begin{array}{l}94(92) \\
96(94)\end{array}$ & $\begin{array}{l}8(8) \\
6(6)\end{array}$ & $\begin{array}{l}0(0) \\
0(0)\end{array}$ & $\begin{array}{l}102(100) \\
102(100)\end{array}$ & 0,625 \\
\hline $\begin{array}{l}\text { Grau-Weiß- } \\
\text { Differenzierung } \\
\text { des } \\
\text { zentralen Kortex }\end{array}$ & $\begin{array}{l}\text { FDCT } \\
\text { MDCT }\end{array}$ & $\begin{array}{l}98(96) \\
99(97)\end{array}$ & $\begin{array}{l}4(4) \\
3(3)\end{array}$ & $\begin{array}{l}0(0) \\
0(0)\end{array}$ & $\begin{array}{l}102(100) \\
102(100)\end{array}$ & 0,813 \\
\hline $\begin{array}{l}\text { Grau-Weiß- } \\
\text { Differenzierung } \\
\text { des Zerebellums }\end{array}$ & $\begin{array}{l}\text { FDCT } \\
\text { MDCT }\end{array}$ & $\begin{array}{l}57(56) \\
88(86)\end{array}$ & $\begin{array}{l}37(36) \\
13(13)\end{array}$ & $\begin{array}{l}8(8) \\
1(1)\end{array}$ & $\begin{array}{l}102(100) \\
102(100)\end{array}$ & $<0,001$ \\
\hline
\end{tabular}

FDCT: Flachdetektor Computertomographie, MDCT: Multidetektor Computertomographie. Die jeweiligen Prozentangaben für „n“ beziehen sich auf die Kollektivgröße von 102 Patient*innen.

Insgesamt erfolgte die Ischämiedetektion mittels Aufnahmen der FDCT mit einer Sensitivität von $71 \%$ und einer Spezifität von 94\% ( $<<0,001$; AUC 0,83; KI 0,74 bis 0,89). Die von den Auswerter*innen gemessenen ASPECTS mit der FDCT (vgl. Abbildung 2) zeigten eine mittlere Abweichung von 0,5 (95\% KI 0,12 bis 0,88) verglichen mit den MDCT-Daten.

Auch die subjektive Bewertung der Auswerter*innen bezüglich der Detektion ischämischer Läsionen zeigte für große Läsion mit einem ASPECTS von 0-4 Punkten, dass in 98\% der FDCTFälle und in 99\% der MDCT-Fälle ein sicherer Ausschluss oder eine sichere Bestätigung einer solchen Läsion möglich gewesen war. 


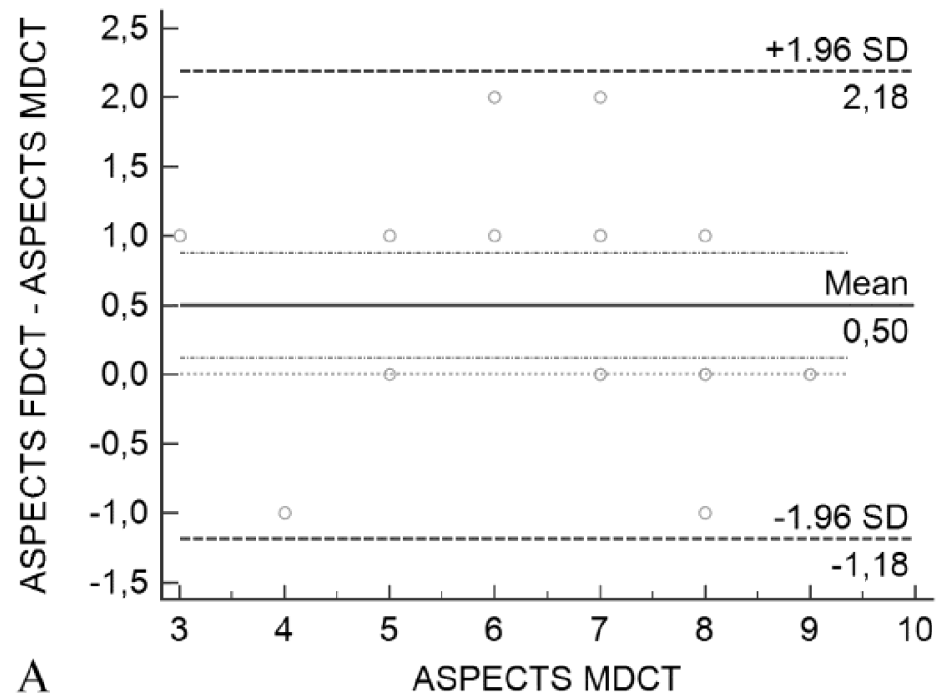

Abbildung 2: Bland Altman Plot der von den Auswertenden gemessenen ASPECTS mittels FDCT und MDCT im bimodalen Vergleich, entnommen aus (Leyhe et al. 2017)(CC BY 4.0).

Die Auswertung der Daten bezüglich der Blutungsdetektion zeigte zunächst für die Subarachnoidalblutungen (SAB), dass mit der Goldstandard-Methode der MDCT bei 39 Patienten eine SAB vorlag. Mit der FDCT konnten 37 dieser 39 Blutungen ebenfalls erkannt werden, zwei Blutungen wurden falsch positiv und weitere zwei Blutungen falsch negativ erkannt (Siehe Tabelle 4). Bezüglich der Unterteilung anhand der gemessenen Schichten (siehe Einteilung Kapitel 2.4) handelte es sich bei den falsch negativ gemessenen Blutungen um eine kleine Subarachnoidalblutung (siehe 2.4 "Analyse der Bildgebung und Statistik“: 0-25. Perzentile entspricht „klein“) und um eine mittelgroße Blutung (25.- 75. Perzentile). Die Detektion der Subarachnoidalblutungen anhand von FDCT-Aufnahmen erfolgte somit mit einer Sensitivität von 95\% und einer Spezifität von 97\% ( $\mathrm{p}<0,001$; AUC 0,96; 95\% KI 0,89 bis 0,98; siehe Tabelle 4: Blutungsdetektion).

Im untersuchten Patient*innenkollektiv konnte bei 34 Patienten*innen eine intraventrikuläre Blutung diagnostiziert werden. Bei den FDCT-Aufnahmen wurde eine Blutung falsch negativ erkannt, bei der es sich um eine kleine Blutung (0-25. Perzentile) handelte. Die Auswertung der FDCT- Aufnahmen erfolgte mit einer Sensitivität von 97\% und einer Spezifität von $100 \%$ ( $\mathrm{p}<$ 0,001; AUC 0,98; 95\% KI 0,93 bis 0,99; siehe Tabelle 4).

Insgesamt konnten alle intraparenchymalen Blutungen anhand von FDCT- und MDCTAufnahmen erkannt werden, somit erfolgte deren Analyse mit einer Sensitivität von 100\% und einer Spezifität von 99\% ( $\mathrm{p}<0,001$; AUC 0,99; 95\% KI 0,95 bis 1,00; siehe Tabelle 4). 
Die Bildqualität bezüglich der Blutungsdetektion unterschied sich für den supra-und den infratentoriellen Bereich: Eine ,sehr gute“ Bildqualität für supratentorielle Schichten wurde für $87 \%$ der FDCT-Serien bewertet (vgl. bei 93\% der MDCT-Serien, $\mathrm{p}=0,266$ ). Für die infratentorielle Bildgebung wurde die Qualität in 58\% der FDCT-Aufnahmen als „sehr gut“ bewertet, was sich als signifikanter Unterschied im Vergleich zu der Bewertung der MDCTAufnahmen zeigte (vgl. bei 78\% der MDCT-Aufnahmen; $\mathrm{p}=0,002)$.

Die subjektiven Angaben über die Blutungsdiagnostik zeigten, dass die Auswerter bei 98\% der FDCT-Aufnahmen und bei 99\% der MDCT-Aufnahmen eine sichere Aussage treffen konnten, ob eine Blutung vorläge $(p=1)$.

Tabelle 4: Blutungsdetektion

\begin{tabular}{|l|l|l|l|l|l|}
\hline \multicolumn{2}{|l|}{} & MDCT (-) & MDCT (+) & Sensitivität & Spezifität \\
\hline \multirow{2}{*}{ SAB } & FDCT (-) & 61 & 2 & $95 \%$ & \multirow{2}{*}{$97 \%$} \\
\cline { 2 - 4 } & FDCT (+) & 2 & 37 & & \\
\hline IVB & FDCT (-) & 68 & 1 & \multirow{2}{*}{$97 \%$} & \multirow{2}{*}{$100 \%$} \\
\cline { 2 - 4 } & FDCT (+) & 0 & 33 & & \\
\hline \multirow{2}{*}{ IPB } & FDCT (-) & 79 & 0 & \multirow{2}{*}{$100 \%$} & \\
\cline { 2 - 4 } & FDCT (+) & 1 & 22 & & \\
\hline
\end{tabular}

FDCT: Flachdetektor Computertomographie, IPB: Intraparenchymale Blutung, IVB: Intraventrikuläre Blutung, MDCT: Multidetektor Computertomographie, SAB: Subarachnoidalblutung. (+): in dieser Modalität detektiert,(-): in dieser Modalität nicht detektiert/ nicht vorhanden.

Die Auswertungen der verschiedenen Artefakttypen zeigt, dass bei 22 der 102 Patient*innen in der FDCT-Bildgebung und bei 15 der 102 Patient*innen in der MDCT-Bildgebung Metallartefakte auftraten. Der Großteil der Artefakte hat einen „sichtbaren“ Einfluss auf die Bilder (FDCT 10 (9,8\%) und MDCT 5 (4,9\%); siehe Tabelle 5), ein Metallartefakt in der FDCT und zwei in der MDCT machten die Auswertung unmöglich. Zwischen den beiden Modalitäten zeigte sich kein statistisch signifikanter Unterschied (siehe Tabelle 5: $\mathrm{p}=0,330$ ). Bewegungsartefakte traten bei 26 Patient*innen in der FDCT-Serie und bei 6 Patient*innen in der MDCT-Serie auf. Am häufigsten (11 mal) trat dieser Artefakttyp in der FDCT als „sichtbar“ 
auf und in der MDCT am häufigsten (vier mal) als „wesentliche Beeinträchtigung“ für die Auswertung.

Streifenartefakte sind insgesamt in beiden Modalitäten am häufigsten zu finden (FDCT vs. MDCT: 74 (72,5\%) vs. 20 (19,6\%); siehe Tabelle 5) und ebenfalls zum Großteil als „sichtbar“ bewertet worden (FDCT vs. MDCT: 49 (48,0\%) vs. 12 (12,7\%); siehe Tabelle 5). Ring- und Einbrandartefakte zeigten sich nur in der FDCT-Bildgebung in jeweils 24 Fällen.

Insgesamt zeigte sich die Bildgebung der FDCT anfälliger für Artefakte. Statistisch signifikante Unterschiede zeigten sich in der Kategorie der Streifenartefakte sowie bei den ausschließlich in der FDCT aufgetretenen Einbrand- und Ringartefakten.

Tabelle 5: Einfluss der verschiedenen Artefakttypen

\begin{tabular}{|c|c|c|c|c|c|c|c|c|}
\hline & & $\begin{array}{c}\text { Kaum } \\
\text { sichtbar }\end{array}$ & $\begin{array}{l}\text { sicht- } \\
\text { bar }\end{array}$ & $\begin{array}{c}\text { Auswertung } \\
\text { wesentlich } \\
\text { beeinträchtigt }\end{array}$ & $\begin{array}{l}\text { Auswertung } \\
\text { unmöglich }\end{array}$ & $\begin{array}{c}\text { Nicht } \\
\text { vorhanden }\end{array}$ & Gesamt & Wilcoxe \\
\hline Variable & & n $(\%)$ & n $(\%)$ & n ( $\%)$ & n (\%) & n $(\%)$ & $\mathbf{n}$ & $\mathrm{p}$ \\
\hline Metallartefakte & $\begin{array}{l}\text { FDCT } \\
\text { MDCT }\end{array}$ & $\begin{array}{l}6(5,9) \\
1(1,0)\end{array}$ & $\begin{array}{l}10 \\
(9,8) \\
5(4,9)\end{array}$ & $\begin{array}{l}5(4,9) \\
7(6,9)\end{array}$ & $\begin{array}{l}1(1,0) \\
2(2,0)\end{array}$ & $\begin{array}{l}80(78,4) \\
87(85,3)\end{array}$ & $\begin{array}{l}22 \\
15\end{array}$ & 0,330 \\
\hline Bewegungsartefakte & $\begin{array}{l}\text { FDCT } \\
\text { MDCT }\end{array}$ & $\begin{array}{l}6(5,9) \\
0(0,0)\end{array}$ & $\begin{array}{l}11 \\
(10,8) \\
2(2,0)\end{array}$ & $\begin{array}{l}9(8,8) \\
4(3,9)\end{array}$ & $\begin{array}{l}0(0,0) \\
0(0,0)\end{array}$ & $\begin{array}{l}76(74,5) \\
96(94,1)\end{array}$ & $\begin{array}{l}26 \\
6\end{array}$ & 0,006 \\
\hline Ringartefakte & $\begin{array}{l}\text { FDCT } \\
\text { MDCT }\end{array}$ & $\begin{array}{l}14(13,7) \\
0(0,0)\end{array}$ & $\begin{array}{l}9(8,8) \\
0(0,0)\end{array}$ & $\begin{array}{l}1(1,0) \\
0(0,0)\end{array}$ & $\begin{array}{l}0(0,0) \\
0(0,0)\end{array}$ & $\begin{array}{l}78(76,5) \\
102(100)\end{array}$ & $\begin{array}{l}24 \\
0\end{array}$ & $<0,0001$ \\
\hline Streifenartefakte & $\begin{array}{l}\text { FDCT } \\
\text { MDCT }\end{array}$ & $\begin{array}{l}15(14,7) \\
5(4,9)\end{array}$ & $\begin{array}{l}49 \\
(48,0) \\
13 \\
(12,7)\end{array}$ & $\begin{array}{l}10(9,8) \\
2(2,0)\end{array}$ & $\begin{array}{l}0(0,0) \\
0(0,0)\end{array}$ & $\begin{array}{l}28(27,5) \\
82(80,4)\end{array}$ & $\begin{array}{l}74 \\
20\end{array}$ & $<0,0001$ \\
\hline Einbrandartefakte & $\begin{array}{l}\text { FDCT } \\
\text { MDCT }\end{array}$ & $\begin{array}{l}6(5,9) \\
0(0,0)\end{array}$ & $\begin{array}{l}18 \\
(17,6) \\
0(0,0)\end{array}$ & $\begin{array}{l}0(0,0) \\
0(0,0)\end{array}$ & $\begin{array}{l}0(0,0) \\
0(0,0)\end{array}$ & $\begin{array}{l}78(76,5) \\
102(100)\end{array}$ & $\begin{array}{l}24 \\
0\end{array}$ & $<0,0001$ \\
\hline Gesamt & $\begin{array}{l}\text { FDCT } \\
\text { MDCT }\end{array}$ & $\begin{array}{l}47 \\
6\end{array}$ & $\begin{array}{l}87 \\
20\end{array}$ & $\begin{array}{l}25 \\
13\end{array}$ & $\begin{array}{l}1 \\
2\end{array}$ & $\begin{array}{l}340 \\
469\end{array}$ & & \\
\hline
\end{tabular}

Übersicht über Häufigkeit und Einfluss auf die Bildqualität und Auswertung durch verschiedene Artefakttypen. Die Größe für „n“ bzw. die dazugehörige Prozentzahl bezieht sich auf die in der Tabelle aufgeführte Gesamtzahl des jeweiligen Artefakts. 


\section{Diskussion}

Diese Dissertation beschäftigt sich mit der neuen Generation der Flachdetektor-CT und deren Anwendung im Rahmen der Schlaganfalldiagnostik und -therapie im Vergleich zur Goldstandard-Methode, der Multidetektor-CT. Die bisherigen diagnostischen Einsatzmöglichkeiten der Flachdetektor-Computertomographie bewegten sich im Wesentlichen im Bereich der postinterventionellen Komplikationsanalyse wie beispielsweise zum Ausschluss einer im Rahmen der Behandlung aufgetretenen Blutung (Heran et al. 2006). Dies hatte in der Vergangenheit bereits zu einer Vereinfachung des Behandlungsablaufs geführt, da ein erneuter Transport zurück zur MDCT für die abschließende Bildgebung vermieden werden konnte. Ein breiterer diagnostischer Einsatz fand aufgrund der technischen Limitationen früherer Generationen vor allem im infratentoriellen Bereich und bezüglich der Detektion von Ischämien bisher nicht statt (Struffert et al. 2009; Psychogios et al. 2010). Eine weitere Limitation des Einsatzes der Flachdetektor-CT entstand durch die erhöhte Anfälligkeit gegenüber Bildartefakten (Struffert et al. 2009).

So ließ sich die FDCT bislang nicht als vorrangiges bildgebendes Verfahren etablieren, insbesondere da durch die breite Anwendung einer systemischen Thrombolysetherapie mit rekombinantem tissue plasminogen activator (rtPA) der sichere Ausschluss einer intrakraniellen Blutung gewährleistet sein muss.

Mit der neuen Generation der Flachdetektor-CT könnte aufgrund des neu entwickelten Flachdetektors eine verbesserte Grau-Weiß-Differenzierung ähnlich der einer MDCT erreicht werden. Darüber hinaus konnten andere technische Bedingungen verändert werden. So wurden Spannungsschwankungen verringert und eine erhöhte Durchleuchtungskraft durch den Einsatz einer neuen Röntgenröhre erreicht.

Wie der aktuellen Studienlage entsprechend konnten auch in dieser Studie eine hervorragende Darstellung des supratentoriellen Ventrikelsystems (100\% „gut beurteilbar“ für FDCT und MDCT) und der Subarachnoidalräume festgestellt (90\%, gut beurteilbar“ für FDCT und 96\% für MDCT) und insgesamt keine statistische Differenz zwischen beiden Modalitäten gezeigt werden. Im Gegensatz zu den vorherigen Analysen wurde nun das infratentorielle Ventrikelsystem zu 90\% als diagnostisch beurteilbar eingestuft (vgl. mit früheren Studien: 60,7\% „gutbeurteilbar/diagnostisch“ (Psychogios et al. 2010)), der infratentorielle Subarachnoidalraum zu 74\% (vgl. mit früheren Studien: 28,6\% „gut beurteilbar/ diagnostisch“ (Psychogios et al. 2010)). Unsere Auswertung der Ischämiediagnostik zeigte eine gute Korrelation beider Modalitäten bezüglich des ASPECTS ( $\mathrm{r}=0,78,95 \%$ CI 0,54-0,90). 
Dies zeigt sich kohärent mit einer weiteren Arbeit der Stroke Research Group Göttingen, welche ebenfalls keinen signifikanten Unterschied zwischen den mittels FDCT gemessenen und dem mittels MDCT gemessenen ASPECTS feststellen konnte (Maier et al. 2018).

Bezüglich unserer Auswertung der einzelnen Parameter zeigten sich neben den aufgeführten Ergebnissen noch Limitationen hinsichtlich der diagnostischen Möglichkeiten: So ist auch bei der neuen Generation der FDCT die Evaluation im infratentoriellen Subarachnoidalraum mit einer diagnostischen Genauigkeit von 74\% (vgl.

Tabelle 2) immer noch der MDCT signifikant unterlegen. Dies könnte vor allem die Detektion kleiner infratentorieller Subarachnoidalblutungen beeinflussen.

Unsere retrospektive Analyse des Einflusses verschiedener Artefakttypen auf die Auswertbarkeit der Bilder zeigt, dass bezüglich des Ausmaßes des Einflusses der Artefakte sich der überwiegende Teil in den Kategorien „kaum sichtbar“ und „sichtbar“ befindet (vgl. Tabelle 5). Die Kategorie „machen die Auswertung unmöglich“ wird einmal für die FDCT-Bildgebung und zweimal für die MDCT-Bildgebung ausgewertet (beides in der Gruppe der Metallartefakte).

Durch den Einsatz neuer Rekonstruktionsalgorithmen, wie beispielsweise der MetallartefaktReduktionsalgorithmus („MARA“), kann der Einfluss der Artefakte auf die Bildqualität verringert werden (Pjontek et al. 2016).

In unserem Patientenkollektiv entfiel der größte Anteil bezüglich der Metallartefakte in die Kategorie „sichtbar“ (vgl. Tabelle 5: FDCT 10 (9,8\%) und MDCT 5 (4,9\%)). Wie bereits zuvor erwähnt, machen lediglich Metallartefakte (vgl. Tabelle 5) die Auswertung einer Bildgebung unmöglich im Gegensatz zu den anderen untersuchten Artefakttypen.

Eine Beschäftigung mit den Einbrandartefakten, welche sich nur in der FDCT-Bildgebung zeigten (vgl. Tabelle 5), ergab in Rücksprache mit dem technischen Team des Herstellers, dass diese meist auftreten, wenn zuvor eine zeitintensive Intervention stattfand. Sie lassen sich reduzieren, indem das Prozedere der Bildgebung verändert und die besagte Serie mit einem kurzen Abstand zur Intervention (beispielsweise nach dem Einbringen des Verschlusssystems) aufgenommen wird.

Die Streifen- und viele der Bewegungsartefakte lassen sich in Rücksprache mit dem technischen Team des Herstellers auf die Kopfschale in der Angiographie zurückführen. Die Kopfschale setzt sich aus dichten Kohlefasern zusammen, welche sich als Artefakte in der Bildgebung manifestieren können. Durch den Einsatz einer neuen Kopfschale könnten sich diese Artefakte reduzieren lassen. Die Korrektur der Bewegungsartefakte könnte sich durch eine andere Kopfschale, welche den Patienten sicher in der Fixierung halten könnte, ebenfalls verbessern. 
$\mathrm{Zu}$ den Limitationen dieser Studie zählt zunächst der retrospektive Charakter der Analyse. Die zeitliche Differenz zwischen den beiden Aufnahmen könnte dazu geführt haben, dass eventuelle Veränderungen, wie beispielsweise eine postinterventionelle Blutung, auch erst auf den zu einem späteren Zeitpunkt nach der Intervention entstandenen MDCT-Aufnahmen erkannt werden konnten und nicht bereits auf der vorherigen peri-interventionellen Aufnahme. In unserer Auswertung der Studienergebnisse zeigte sich, dass es sich bei den beiden falsch positiv detektierten Subarachnoidalblutungen (vgl. Tabelle 4) um eine Kontrastmittelextravasation handelte, die sich bei den zu einem späteren Zeitpunkt aufgenommenen MDCT-Aufnahmen aufgelöst hatte.

Die diagnostischen Anforderungen im Rahmen der Schlaganfalltherapie haben sich seit 2015 zunehmend verändert: Die Wichtigkeit einer genauen Detektion ischämischer Frühzeichen im CT hingegen tritt im Vergleich eher in den Hintergrund, da die aktuelle Studienlage sich mehr auf den Gefäßstatus bezüglich einer möglichen Versorgung des betroffenen Gebietes durch Kollateralgefäße konzentriert und sich eine Empfehlung bezüglich der Therapie mittels Thrombektomie auch bei größeren Ischämien (ASPECTS 0-5 Punkte) abzeichnet (Goyal et al. 2016; Tan et al. 2016; Schnieder et al. 2019).

Zentrale Bedeutung für das therapeutische Konzept besitzt hingegen der sichere Ausschluss einer intrakraniellen Blutung, um eine Thrombolysetherapie mit rtPA einleiten zu können. Die neue Generation der FDCT zeigte für die Detektion der intraparenchymalen Blutung (IPB), intraventrikuläre Blutung (IVB) und für die subarachnoidale Blutung (SAB) insgesamt eine hohe Sensitivität und Spezifität von jeweils $>95 \%$ (vgl. Tabelle 4). Neben den bereits aufgeführten zwei falsch positiven Subarachnoidalblutungen aufgrund einer Kontrastmittelextravasation, ließen sich zwei falsch negative Ergebnisse auf starke Bewegungsartefakte zurückführen, welche die Blutung auch in der MDCT nur in wenigen Schichten auswertbar machten. Alle intraparenchymalen Blutungen konnten mittels FDCT und MDCT erkannt werden. Somit kann der für den Beginn der Thrombolysetherapie entscheidende Blutungsausschluss auch mithilfe der FDCT gestellt werden.

Dies kann als Ausblick in Richtung eines so genannten one-stop-management-Konzepts dienen: Dieses Konzept sieht eine Kombination von Diagnostik und Therapie an der FlachdetektorCT unter Auslassen der Multidetektor-CT als neuen Goldstandard für die Schlaganfalldiagnostik und -therapie vor.

Eine Umstrukturierung des zeitlichen Ablaufs in der Schlaganfalltherapie zugunsten kürzerer Prozesszeiten als wesentlicher Faktor bezüglich des Outcomes der einzelnen Patientinnen und Patienten wird international immer stärker diskutiert. 
So zeigte eine weitere Auswertung der in der Einleitung aufgeführten, multizentrische Studien bezüglich der Überlegenheit der mechanischen Thrombektomie, einen direkten Zusammenhang zwischen der Länge von Diagnostik und Therapie und dem klinischen Outcome der Betroffenen auf (Fransen et al. 2016; Saver et al. 2016). Häufig wird eine Richtgröße von einer Zunahme der durch den Schlaganfall bedingten Beeinträchtigungen von bis zu 15\% pro 30 Minuten nach Beginn der Symptome angegeben (Mazighi et al. 2013; Mendez et al. 2018).

Es wird ein „klar strukturierter Ablauf mit festen Zuständigkeiten empfohlen wobei als Gradmesser bestimmte Zeitspannen wie die door-to-groin oder die door-to-reperfusion dienen können.“ (Menon et al. 2014; McTaggart et al. 2017). Neben der Wichtigkeit eines raschen Transportes in ein Krankenhaus, welches über die Möglichkeiten einer interdisziplinären Schlaganfallversorgung verfügt, lassen sich verschiedene Faktoren innerhalb eines Krankenhauses identifizieren, welche zu Behandlungsverzögerungen führen können. Neben einer klaren Struktur wird die Notwendigkeit einer raschen Mitbetreuung der Betroffenen von Seiten der Anästhesie betont, sowie eine effiziente Bildgebung mit raschem Therapiebeginn, welcher nicht durch häufiges Umlagern, Unklarheiten bezüglich weiterer Bildgebungsmöglichkeiten wie die Notwendigkeit einer CT-Perfusion, etc. verzögert werden sollte (Goyal et al. 2014).

Bereits nach Implementierung einer interdisziplinären SOP( Standard Operating Procedure) für den Ablauf der Schlaganfalltherapie an der UMG in Zusammenarbeit mit der Klinik für Neurologie und der Klinik für Anästhesiologie konnte durch strukturelle Veränderungen und klare zeitliche Vorgaben eine Reduzierung der Zeiten erreicht werden (Schregel et al. 2016). Vor allem die Zeitspanne zwischen dem Symptombeginn und der Wiedereröffnung des Gefäßes konnte von einer medianen Zeit von 264 Minuten auf 211 Minuten reduziert werden, was die Autoren unter anderem auf eine schnelle Bereitstellung der Bildgebung zurückführten. Auch die so genannte door-to-groin-time konnte von initial >120 Minuten auf ca. 60 Minuten reduziert werden. Dies führte zu einer Verbesserung des klinischen Outcomes gemessen anhand des modified Rankin Scale (mRS) mit einer Zunahme innerhalb der Gruppe der Patient*innen ohne Defizite nach einem Schlaganfall ( $\mathrm{mRS}=0$ ) sowie einer Abnahme innerhalb der Gruppe schwerst betroffener Patient*innen (mRS = 4 oder 5) (Schregel et al. 2016). Auch andere Arbeitsgruppen konnten durch eine Umstrukturierung der innerklinischen Abläufe eine Verkürzung des Behandlungsprozesses erreichen: Das so genannte Helsinki stroke model, welches unter anderem in Finnland, Australien und Neuseeland klinisch erprobt wurde, betont als Basis für eine effiziente Therapie eine umfassende Vorbereitung nach Ankündigung eines Patienten oder 
Patientin, um dann mit klarer Verantwortung und möglichst wenig Beteiligten eine zügige Diagnostik und Therapie durchführen zu können (Meretoja et al. 2012; Meretoja et al. 2013).

Eine weitere zeitliche Reduktion könnte somit vor allem im Bereich der Bildgebung durch die oben beschriebene Neustrukturierung möglich sein. Durch eine Zusammenführung von Diagnostik und Therapie in einem Raum wird der Ablauf vereinfacht und zeitlich verkürzt. Ein erster Case Report unserer Abteilung im März 2017 zeigt, dass die Zeit zwischen Ankunft im Angiographie-Raum bis zur Leistenpunktion auf 15 Minuten und die door-to-groin-time insgesamt auf 23 Minuten (die leitliniengemäß vorgegebene Zeit liegt bei 60 Minuten (DGN 2012)) verkürzt werden konnte (Psychogios M-N et al. 2017b). In einer weiteren Untersuchung mit einer Kohorte von 30 Patient*innen, welche im Zeitraum von Juni bis Dezember 2016 in der UMG mit einem ischämischen Schlaganfall diagnostiziert und therapiert wurden, konnten ebenfalls signifikante zeitliche Unterschiede festgestellt werden: die door-to-groin-time wurde auf diese Weise von 54,5 Minuten (in der Kontrollgruppe, welche Anfang 2016 noch eine Bildgebung an beiden Geräten erhielten) auf 20,5 Minuten (one-stop-management-Gruppe) gesenkt (Psychogios et al. 2017). Diese Ergebnisse zeigten sich im Einklang mit weiteren Kliniken, welche einen one-stop-management- Arbeitsablauf für die Schlaganfallbehandlung etablierten (Jadhav et al. 2017; Mendez et al. 2018).

Eine genauere Evaluation des klinischen Outcomes beziehungsweise eine Implementierung und deren Folgen in anderen Krankenhäusern sind hierbei Fragestellungen für weitere prospektive Untersuchungen. 


\section{$5 \quad$ Zusammenfassung}

In der vorliegenden Arbeit wurde die Anwendbarkeit der neusten Generation der FlachdetektorComputertomographie bezüglich der Detektion von hämorrhagischen und ischämischen Läsionen im Vergleich zur Goldstandarduntersuchung, der MultidetektorComputertomographie, untersucht. Insgesamt wurden die Bilddaten von 102 Patient*innen anonymisiert von drei unterschiedlich erfahrenen Auswerter*innen analysiert und deren Ergebnisse statistisch ausgewertet.

Es konnte gezeigt werden, dass die neuste Generation der Flachdetektor-Computertomographie bezüglich der Bewertung des Ventrikelsystems, der Subarachnoidalräume und der GrauWeißDifferenzierung der Basalganglien, der Inselrinde und des Zentralcortex keinen signifikanten Unterschied zur Goldstandardmethode Multidetektor-Computertomographie aufweist. Ein Unterschied zeigte sich bezüglich der Grau-Weiß-Differenzierung des Cerebellums. Die Blutungsdetektion von Subarachnoidalblutungen, intraventrikulären und intraparenchymalen Blutungen erfolgte mit einer Sensitivität und Spezifität von mindestens 94\%. Der Einfluss der Artefakte zeigte einen statistisch signifikanten Unterschied zwischen beiden Modalitäten mit Ausnahme der Metallartefakte. Insgesamt wurde die Mehrzahl der Artefakte als „,kaum sichtbar“ oder ,sichtbar“" gewertet.

Als Ausblick können die Ergebnisse auch Auswirkungen auf den klinischen Alltag der Schlaganfalldiagnostik und -therapie haben. So ist mit der neusten Generation der Flachdetektor-Computertomographie eine zuverlässige Detektion hämorrhagischer und ischämischer Läsionen möglich, allerdings zeigen sich noch Einschränkungen im Bereich des Cerebellums und durch den Einfluss verschiedener Artefakte. Zukünftig kann die Flachdetektor-Computertomographie im Rahmen des one-stop-management-Diagnostik und Therapie in einem Gerät vereinen und somit eine Auswirkung auf die Behandlungszeit und perspektivisch auch auf das klinische Outcome von Schlaganfallpatient*innen haben.

\section{$6 \quad$ Literaturverzeichnis}

Abstracts: 51. Jahrestagung der Deutschen Gesellschaft für Neuroradiologie e.V. in Kooperation mit der 24. Jahrestagung der Österreichischen Gesellschaft für Neuroradiologie, 5.-8. Oktober 2016, Gürzenich, Köln (2016) Clin Neuroradiol 26, 1-111 
Albers GW, Caplan LR, Easton JD, Fayad PB, Mohr JP, Saver JL, Sherman DG (2002): Transient Ischemic Attack — Proposal for a New Definition. N Engl J Med 347, 1713-1716

Benjamin EJ, Blaha MJ, Chiuve SE, Cushman M, Das SR, Deo R, de Ferranti SD, Floyd J, Fornage M, Gillespie C, et al. (2017): Heart Disease and Stroke Statistics-2017 Update: A Report From the American Heart Association. Circulation 135, e146-e603

Berkhemer OA, Fransen PSS, Beumer D, van den Berg LA, Lingsma HF, Yoo AJ, Schonewille WJ, Vos JA, Nederkoorn PJ, Wermer MJH, et al. (2015): A Randomized Trial of Intraarterial Treatment for Acute Ischemic Stroke. N Engl J Med 372, 11-20

Broderick JP, Palesch YY, Demchuk AM, Yeatts SD, Khatri P, Hill MD, Jauch EC, Jovin TG, Yan B, Silver FL, et al. (2013): Endovascular therapy after intravenous t-PA versus t-PA alone for stroke. $\mathrm{N}$ Engl J Med $\underline{368}, 893-903$

Campbell BCV, Mitchell PJ, Kleinig TJ, Dewey HM, Churilov L, Yassi N, Yan B, Dowling RJ, Parsons MW, Oxley TJ, et al. (2015): Endovascular Therapy for Ischemic Stroke with Perfusion-Imaging Selection. N Engl J Med $\underline{372}, 1009-1018$

DGN (2012): Akuttherapie des ischämischen Schlaganfalls. S1 - Leitlinie für Diagnostik und Therapie in der Neurologie. Hrsg. v. der Deutschen Gesellschaft für Neurologie. Auflage 5, September 2012.

https://www.dgn.org/images/red_leitlinien/LL_2012/pdf/11_22_2012_akuttherapie_des_ischmi schen_schlaganfalls_update.pdf abgerufen am 26.06.2019.

DGN (2015): Akuttherapie des ischämischen Schlaganfalls- Ergänzung 2015 - Rekanalisierende Therapie - -S2k- Leitlinien für Diagnostik und Therapie in der Neurologie. Hrsg. v. der Deutschen Gesellschaft für Neurologie. 06.10.2015. https://www.dgn.org/images/red_leitlinien/LL_2015/PDFs_Download/030140_LL_akuter-ischaemischer-schlaganfall_final.pdf. Abgerufen am 26.06.2019.

Doerfler A, Gölitz P, Engelhorn T, Kloska S, Struffert T (2015): Flat-Panel Computed Tomography (DYNA-CT) in Neuroradiology. From High-Resolution Imaging of Implants to One-StopShopping for Acute Stroke. Clin Neuroradiol 25, 291-297

Easton, Saver Jeffrey L., Albers Gregory W., Alberts Mark J., Chaturvedi Seemant, Feldmann Edward, Hatsukami Thomas S., Higashida Randall T., Johnston S. Claiborne, Kidwell Chelsea S., et al. (2009): Definition and Evaluation of Transient Ischemic Attack. Stroke 40, 2276-2293

Emberson J, Lees KR, Lyden P, Blackwell L, Albers G, Bluhmki E, Brott T, Cohen G, Davis S, Donnan G, et al. (2014): Effect of treatment delay, age, and stroke severity on the effects of intravenous thrombolysis with alteplase for acute ischaemic stroke: a meta-analysis of individual patient data from randomised trials. Lancet Lond Engl $\underline{384}, 1929-1935$ 
Fransen PSS, Berkhemer OA, Lingsma HF, Beumer D, van den Berg LA, Yoo AJ, Schonewille WJ, Vos JA, Nederkoorn PJ, Wermer MJH, et al. (2016): Time to Reperfusion and Treatment Effect for Acute Ischemic Stroke: A Randomized Clinical Trial. JAMA Neurol 73, 190-196

Gomez CR, Orr SC, Soto RD (2002): Neuroendovascular rescue: Interventional treatment of acute ischemic stroke. Curr Treat Options Cardiovasc Med 4, 405-419

Goyal, Menon Bijoy K., Hill Michael D., Demchuk Andrew (2014): Consistently Achieving Computed Tomography to Endovascular Recanalization <90 Minutes. Stroke 45, e252-e256

Goyal M, Demchuk AM, Menon BK, Eesa M, Rempel JL, Thornton J, Roy D, Jovin TG, Willinsky RA, Sapkota BL, et al. (2015): Randomized Assessment of Rapid Endovascular Treatment of Ischemic Stroke. N Engl J Med 372, 1019-1030

Goyal M, Menon BK, van Zwam WH, Dippel DWJ, Mitchell PJ, Demchuk AM, Dávalos A, Majoie CBLM, van der Lugt A, de Miquel MA, et al. (2016): Endovascular thrombectomy after largevessel ischaemic stroke: a meta-analysis of individual patient data from five randomised trials. The Lancet $\underline{387}, 1723-1731$

Grillenberger A, Fritsch E: Computertomographie: Einführung in ein modernes bildgebendes Verfahren. 2., aktualisierte Aufl; Facultas.wuv, Wien 2012

Heran NS, Song JK, Namba K, Smith W, Niimi Y, Berenstein A (2006): The Utility of DynaCT in Neuroendovascular Procedures. Am J Neuroradiol 27, 330-332

Hosten N, Liebig T: Computertomographie von Kopf und Wirbelsäule: 21 Tabellen (Referenz-Reihe radiologische Diagnostik). Georg Thieme Verlag, Stuttgart New York 2000

Hufschmidt A, Lücking CH, Rauer S, Glocker FX, Amtage F (Hrsg.): Neurologie compact: für Klinik und Praxis. 7., überarbeitete und erweiterte Auflage; Georg Thieme Verlag, Stuttgart New York 2017

Jadhav, Kenmuir Cynthia L., Aghaebrahim Amin, Limaye Kaustubh, Wechsler Lawrence R., Hammer Maxim D., Starr Matthew T., Molyneaux Bradley J., Rocha Marcelo, Guyette Francis X., et al. (2017): Interfacility Transfer Directly to the Neuroangiography Suite in Acute Ischemic Stroke Patients Undergoing Thrombectomy. Stroke $\underline{48}, 1884-1889$

Kau T, Hauser M, Obmann SM, Niedermayer M, Weber JR, Hausegger KA (2014): Flat Detector Angio-CT following Intra-Arterial Therapy of Acute Ischemic Stroke: Identification of Hemorrhage

and Distinction from Contrast Accumulation due to Blood-Brain Barrier Disruption. Am J Neuroradiol $\underline{35}$, 1759-1764 
Kramme R (Hrsg.): Medizintechnik: Verfahren - Systeme - Informationsverarbeitung (Springer Reference Technik). 5., vollständig überarbeitete und erweiterte Auflage; Springer, Berlin 2017

Kurz MW, Kurz KD, Farbe E (2013): Acute ischemic stroke - from symptom recognition to thrombolysis. Acta Neurol Scand 127, 57-64

Kyriakou Y, Struffert T, Dörfler A, Kalender WA (2009): Grundlagen der Flachdetektor-CT (FD-CT). Radiol 49, 811-819

Leary MC, Saver JL, Gobin YP, Jahan R, Duckwiler GR, Vinuela F, Kidwell CS, Frazee J, Starkman S (2003): Beyond tissue plasminogen activator: Mechanical intervention in acute stroke. Ann Emerg Med $41,838-846$

Leyhe JR, Tsogkas I, Hesse AC, Behme D, Schregel K, Papageorgiou I, Liman J, Knauth M, Psychogios M-N (2017): Latest generation of flat detector CT as a peri-interventional diagnostic tool: a comparative study with multidetector CT. J Neurointerventional Surg 9, 1253-1257

Liebeskind DS, Tomsick TA, Foster LD, Yeatts SD, Carrozzella J, Demchuk AM, Jovin TG, Khatri P, von Kummer R, Sugg RM, et al. (2014): Collaterals at angiography and outcomes in the Interventional Management of Stroke (IMS) III trial. Stroke 45, 759-764

Maier IL, Bauerle M, Kermer P, Helms H-J, Buettner T (2013): Risk prediction of very early recurrence, death and progression after acute ischaemic stroke. Eur J Neurol 20, 599-604

Maier IL, Leyhe JR, Tsogkas I, Behme D, Schregel K, Knauth M, Schnieder M, Liman J, Psychogios M-N (2018): Diagnosing Early Ischemic Changes with the Latest-Generation Flat Detector CT: A Comparative Study with Multidetector CT. Am J Neuroradiol 39, 881-886

Mazighi, Chaudhry Saqib A., Ribo Marc, Khatri Pooja, Skoloudik David, Mokin Maxim, Labreuche Julien, Meseguer Elena, Yeatts Sharon D., Siddiqui Adnan H., et al. (2013): Impact of Onset-toReperfusion Time on Stroke Mortality. Circulation 127, 1980-1985

McTaggart RA, Ansari SA, Goyal M, Abruzzo TA, Albani B, Arthur AJ, Alexander MJ, Albuquerque FC, Baxter B, Bulsara KR, et al. (2017): Initial hospital management of patients with emergent large vessel occlusion (ELVO): report of the standards and guidelines committee of the Society of NeuroInterventional Surgery. J NeuroInterventional Surg $\underline{9}$, 316-323

Mendez, Requena Manuel, Aires Ana, Martins Nuno, Boned Sandra, Rubiera Marta, Tomasello Alejandro, Coscojuela Pilar, Muchada Marián, Rodríguez-Luna David, et al. (2018): Direct Transfer to Angio-Suite to Reduce Workflow Times and Increase Favorable Clinical Outcome. Stroke $\underline{49}, 2723-2727$

Menon, Almekhlafi Mohammed A., Pereira Vitor Mendes, Gralla Jan, Bonafe Alain, Davalos Antoni, Chapot Rene, Goyal Mayank (2014): Optimal Workflow and Process-Based Performance Measures for Endovascular Therapy in Acute Ischemic Stroke. Stroke 45, 2024-2029 
Meretoja A, Strbian D, Mustanoja S, Tatlisumak T, Lindsberg PJ, Kaste M (2012): Reducing in-hospital delay to 20 minutes in stroke thrombolysis. Neurology $\underline{79}$, 306-313

Meretoja A, Weir L, Ugalde M, Yassi N, Yan B, Hand P, Truesdale M, Davis SM, Campbell BCV (2013): Helsinki model cut stroke thrombolysis delays to 25 minutes in Melbourne in only 4 months. Neurology $\underline{81}, 1071-1076$

Nogueira RG, Jadhav AP, Haussen DC, Bonafe A, Budzik RF, Bhuva P, Yavagal DR, Ribo M, Cognard C, Hanel RA, et al. (2017): Thrombectomy 6 to 24 Hours after Stroke with a Mismatch between Deficit and Infarct. N Engl J Med $\underline{378}, 11-21$

Pexman JHW, Barber PA, Hill MD, Sevick RJ, Demchuk AM, Hudon ME, Hu WY, Buchan AM (2001): Use of the Alberta Stroke Program Early CT Score (ASPECTS) for Assessing CT Scans in Patients with Acute Stroke. Am J Neuroradiol 22, 1534-1542

Pjontek R, Önenköprülü B, Scholz B, Kyriakou Y, Schubert GA, Nikoubashman O, Othman A, Wiesmann M, Brockmann MA (2016): Metal artifact reduction for flat panel detector intravenous CT angiography in patients with intracranial metallic implants after endovascular and surgical treatment. J Neurointerventional Surg $\underline{8}, 824-829$

Psychogios, Behme Daniel, Schregel Katharina, Tsogkas Ioannis, Maier Ilko L., Leyhe Johanna Rosemarie, Zapf Antonia, Tran Julia, Bähr Mathias, Liman Jan, Knauth Michael (2017): One-Stop Management of Acute Stroke Patients. Stroke 48, 3152-3155

Psychogios M-N, Buhk J-H, Schramm P, Xyda A, Mohr A, Knauth M (2010): Feasibility of Angiographic CT in Peri-Interventional Diagnostic Imaging: A Comparative Study with Multidetector CT. Am J Neuroradiol 31, 1226-1231

Psychogios M-N, Knauth M, Bshara R, Schregel K, Tsogkas I, Papageorgiou I, Maier I, Liman J, Behme D (2017a): Computed tomography perfusion-based selection of endovascularly treated acute ischaemic stroke patients - Are there lessons to be learned from the pre-evidence era? Neuroradiol J $\underline{30}, 138-143$

Psychogios M-N, Bähr M, Liman J, Knauth M (2017b): One Stop Management in Acute Stroke: First Mothership Patient Transported Directly to the Angiography Suite. Clin Neuroradiol 27, 389-391

Saver JL, Goyal M, van der Lugt A, Menon BK, Majoie CBLM, Dippel DW, Campbell BC, Nogueira RG, Demchuk AM, Tomasello A, et al. (2016): Time to Treatment With Endovascular Thrombectomy and Outcomes From Ischemic Stroke: A Meta-analysis. JAMA $\underline{316}$, 1279-1289

Schnieder M, Psychogios M, Maier I, Tsogkas I, Schregel K, Kleinknecht A, Knauth M, Bähr M, Liman J, Behme D (2019): The problem of strict image-based inclusion criteria for mechanical thrombectomy - an analysis of stroke patients with an initial low CBV-ASPECTS score. Neuroradiol J $\underline{32}, 287-293$ 
Schregel K, Behme D, Tsogkas I, Knauth M, Maier I, Karch A, Mikolajczyk R, Hinz J, Liman J, Psychogios M-N (2016): Effects of Workflow Optimization in Endovascularly Treated Stroke Patients - A Pre-Post Effectiveness Study. PLOS ONE 11, e0169192 1-12

Schregel K, Tsogkas I, Peter C, Zapf A, Behme D, Schnieder M, Maier IL, Liman J, Knauth M, Psychogios M-N (2018): Outcome Prediction Using Perfusion Parameters and Collateral Scores of Multi-Phase and Single-Phase CT Angiography in Acute Stroke: Need for One, Two, Three, or Thirty Scans? J Stroke 20, 362-372

Schröder, Thomalla G (2017): A Critical Review of Alberta Stroke Program Early CT Score for Evaluation of Acute Stroke Imaging. Front Neurol 1, 1-7

Smith, Sung Gene, Starkman Sidney, Saver Jeffrey L., Kidwell Chelsea S., Gobin Y.Pierre, Lutsep Helmi L., Nesbit Gary M., Grobelny Thomas, Rymer Marilyn M., et al. (2005): Safety and Efficacy of Mechanical Embolectomy in Acute Ischemic Stroke. Stroke 36, 1432-1438

Struffert T, Richter G, Engelhorn T, Doelken M, Goelitz P, Kalender WA, Ganslandt O, Doerfler A (2009): Visualisation of intracerebral haemorrhage with flat-detector CT compared to multislice CT: results in 44 cases. Eur Radiol 19, 619-625

Tan, Wan-Yee Kong, Paliwal Prakash, Gopinathan Anil, Nadarajah Mahendran, Ting Eric, Venketasubramanian Narayanaswamy, Seet Raymond C.S., Chan Bernard P.L., Teoh Hock L., et al. (2016): Good Intracranial Collaterals Trump Poor ASPECTS (Alberta Stroke Program Early CT

Score) for Intravenous Thrombolysis in Anterior Circulation Acute Ischemic Stroke. Stroke $\underline{47}$, 2292-2298

Thomalla G, Audebert H, Berger K, Fiebach J, Fiehler J, Kaps M, Neumann-Haefelin T, Schellinger P, Siebler M, Sobesky J, et al. (2009): Bildgebung beim Schlaganfall - eine Übersicht und Empfehlungen des Kompetenznetzes Schlaganfall. Aktuelle Neurol 36, 354-367

Thomalla G, Simonsen CZ, Boutitie F, Andersen G, Berthezene Y, Cheng B, Cheripelli B, Cho T-H, Fazekas F, Fiehler J, et al. (2018): MRI-Guided Thrombolysis for Stroke with Unknown Time of Onset. N Engl J Med 379, 611-622

Yang, Niu Kai, Wu Yijing, Struffert Tobias, Dorfler Arnd, Schafer Sebastian, Royalty Kevin, Strother Charles, Chen Guang-Hong (2015): Time-Resolved C-Arm Computed Tomographic Angiography Derived From Computed Tomographic Perfusion Acquisition. Stroke 46, 3383-3389 
Publikation

\section{$7 \quad$ Publikation}

Leyhe JR, Tsogkas I, Hesse AC, Behme D, Schregel K, Papageorgiou I, Liman J, Knauth M, Psychogios MN (2017): Latest generation of flat detector CT as a peri-interventional diagnostic tool: a comparative study with multidetector CT. J Neurointerventional Surg $\underline{9}$, 1253-1257 
Publikation

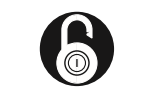

OPEN ACCESS

\section{diagnostic tool: a comparative study with multidetector $\mathrm{CT}$}

\section{ABSTRACT}

Background and purpose Flat detector CT (FDCT) has been used as a peri-interventional diagnostic tool in numerous studies with mixed results regarding image quality and detection of intracranial lesions. We compared the diagnostic aspects of the latest generation FDCT with standard multidetector CT (MDCT). Materials and methods 102 patients were included in our retrospective study. All patients had undergone interventional procedures. FDCT was acquired periinterventionally and compared with postinterventional MDCT regarding depiction of ventricular/subarachnoidal spaces, detection of intracranial hemorrhage, and delineation of ischemic lesions using an ordinal scale. Ischemic lesions were quantified with the Alberta Stroke Program Early CT Scale (ASPECTS) on both examinations. Two neuroradiologists with varying grades of experience and a medical student scored the anonymized images separately, blinded to the clinical history.

Results The two methods were of equal diagnostic value regarding evaluation of the ventricular system and the subarachnoidal spaces.

Subarachnoidal, intraventricular, and parenchymal hemorrhages were detected with a sensitivity of $95 \%, 97 \%$, and $100 \%$ and specificity of $97 \%, 100 \%$ and $99 \%$, respectively, using FDCT. Gray-white differentiation was feasible in the majority of FDCT scans, and ischemic lesions were detected with a sensitivity of $71 \%$ on FDCT, compared with MDCT scans. The mean difference in ASPECTS values on FDCT and MDCT was 0.5 points $(95 \% \mathrm{Cl}$ 0.12 to 0.88 ).

Conclusions The latest generation of FDCT is a reliable and accurate tool for the detection of intracranial hemorrhage. Gray-white differentiation is feasible in the supratentorial region.

Received 8 November 2016 Revised 28 November 2016 Accepted 29 November 2016 Published Online First 20 December 2016

\section{INTRODUCTION}

In the past decade, non-contrast flat detector CT (FDCT), acquired with angiography systems, has been used primarily for postinterventional detection of complications, such as subarachnoidal or parenchymal hemorrhage (PH). ${ }^{1}$ Multiple studies comparing the diagnostic features of FDCT with multidetector CT (MDCT) have shown good image quality in the supratentorial region, with reliable detection of large hemorrhages, albeit limited image quality in the infratentorial region due to diverse image artifacts..$^{2-4}$ Detection of ischemic Leyhe JR, et al. J Neurolntervent Surg 2017;9:1253-1257. doi:10.1136/neurintsurg-2016-01286

Johanna Rosemarie Leyhe, ${ }^{1}$ Ioannis Tsogkas, ${ }^{1}$ Amélie Carolina Hesse, ${ }^{1}$ Daniel Behme, ${ }^{1}$ Katharina Schregel, ${ }^{1}$ Ismini Papageorgiou, ${ }^{1}$ Jan Liman, ${ }^{2}$ Michael Knauth, ${ }^{1}$ Marios-Nikos Psychogios ${ }^{1}$ lesions with FDCT has been inconsistent to date, due to contrast resolution and diverse artifacts of the cone beam acquisition. The latest generation of FDCT promises improved image quality.

The new high dynamic range flat detector with a 16 bit analog-digital conversion in combination with an entire image processing pipeline as well as a reconstruction algorithm chain running on 16 bit allows for enhanced soft tissue resolution. This technology package results in four times more gray value differentiation compared with conventional systems. Improved FDCT fidelity in the detection of hemorrhagic and ischemic lesions could expand the role of FDCT in acute stroke diagnostics (so called 'one stop management') with a possible impact on door to groin times.

In this study, we tested and compared latest generation FDCT images with standard MDCT scans regarding depiction of ventricular/subarachnoidal spaces, detection of ischemic/hemorrhagic lesions, and the presence of artifacts.

\section{METHODS}

We retrospectively screened all angiographic procedures in our department after installation of the latest generation angiography suite (Artis Q Angiography System; Siemens Healthcare GmbH, Forchheim, Germany) from September 2014 to April 2016 for acquisition of FDCT. Patients with FDCT scans were then screened for postinterventional MDCT scans. Only patients with complete FDCT and MDCT scans were included in our analysis. Three scans with marked motion artifacts were excluded. The ethics committee of our hospital waived the need for a formal application or informed patient consent due to the retrospective ign of this study.

FDCT was acquired with a biplane flat detector angiography system using the following parameters: 20 $s$ of rotation; $200^{\circ}$ total angle with approximately 500 projections; $2 \times 2$ binning; $109 \mathrm{kV} ; 1.8 \mu \mathrm{Gy} /$ frame; weighted CT dose index (CTDIw) $\sim 60 \mathrm{mGy}$, effective dose $\sim 2.5 \mathrm{mSv}$. Initial FDCT projections were then reconstructed on a postprocessing workstation (Syngo $X$ Workplace; Siemens Healthcare $\mathrm{GmbH}$ ) with a 'HU smooth' kernel and

'DynaCT Clear' algorithm to images with a $512 \times 512$ matrix. MDCT was acquired on a 128

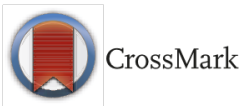

To cite: Leyhe JR, Tsogkas I, Hesse AC, et al. J 
ganglia, insula, central region, and cerebellum. Raters were asked to identify subarachnoidal hemorrhage (SAH), intraventricular hemorrhage (IVH), $\mathrm{PH}$, count the number of slices depicting blood, and measure the dimension of $\mathrm{PH}$. All hemorrhages were divided into three groups with respect to the number of slices containing blood: 0-25 percentile (few), 25-75 percentile (several), and 75-100 percentile (many). In addition, raters were asked to subjectively score image quality for hemorrhage detection using a 5 point ordinal scale (very good; good; mediocre; low; bad). The presence of ischemic lesions was noted, and the Alberta Stroke Program Early CT Scale (ASPECTS) was calculated. Various types of artifacts were documented. Finally, raters were asked if they were confident about the detection/exclusion of a hemorrhage or a large ischemic lesion (ASPECTS 0-4).

Scans were divided into two random groups, consisting of FDCT and MDCT scans, and rated with a 30 day gap in between to minimize recall bias. Window levels were not predefined in order to enhance the detection of ischemic lesions. The initial reconstruction dataset (slice thickness of 0.4 $\mathrm{mm}$ for FDCT and $0.6 \mathrm{~mm}$ for MDCT) was also available and could be used the raters' discretion. Cases of disagreement regarding detection of hemorrhage or ischemia were settled by consensus. Raters were blinded to all clinical information.

Contingency tables and the Wilcoxon test for paired samples were used to compare scores within categories. Categorical variables were compared between groups using Fischer's exact test. Receiver operating characteristic curve analysis was applied to calculate sensitivity and specificity of FDCT for detection of hemorrhage and ischemia compared with MDCT as the gold standard. Measurements of PH as well as ASPECTS ratings on FDCT and MDCT were compared with the Bland-Altman plot. Interobserver agreement was evaluated with Spearman's coefficient of rank correlation. All tests were calculated with the MedCalc statistical package (MedCalc 16.8; MedCalc Software bvba, Ostend, Belgium). The significance level for all tests was set at $\alpha=0.05$. aforementioned postprocessing workstation.

Two neuroradiologists (M$\mathrm{NP},>5$ years of experience; were included in our study (50 women; median age 63.5 years, IQR 53-77).

and a medical student (ACH), Median time between FDCT and MDCT was 4.3 hours (IQR

analyzed all of the images 1.5-6). Patients were examined after coiling, clipping, stenting, thrombectomy, and plain cerebral angiography using a 3 point ordinal scale in $24,1,12,48$, and 17 cases, respectively. FDCT proved to be equivalent to MDCT in the delineation of the $(0=$ not identifiable; supratentorial ventricular system and supratentorial spaces, with $102(100 \%)$ and $98(96 \%)$ 'diagnostic' values, 1=identifiable, but not respectively (table 1). Scores for the infratentorial ventricular system and infratentorial subarachnoidal spaces diagnostic; 2=diagnostic) for on FDCTwere slightly worse, with $92(90 \%)$ and 75 (74\%) scans being scored as 'diagnostic' (figure 1C). However, the following there was no statistically significant difference in the ratings of the ventricular system or subarachnoidal spaces structures/entities: between FDCT and MDCT ( $p=0.106$ and $p=0.177$ ). Gray-white differentiation was feasible on FDCTwith $95 \%, 92 \%$, supratentorial ventricular and $96 \%$ of 'diagnostic' values in the supratentorial region and no statistically significant difference from MDCT system, infratentorial for the basal ganglia, insular cortex, and central region (table 1 ).

ventricular system, Gray-white differentiation on FDCT was limited in the infratentorial region with significantly different scores supratentorial compared with MDCT $(p<0.001)$. Detection of ischemic lesions was feasible on FDCT scans with $71 \%$ sensitivity subarachnoidal space, and $94 \%$ specificity ( $p<0.001$; area under the curve (AUC) $0.83,95 \% \mathrm{Cl} 0.74$ to 0.89 ) compared with MDCT scans. infratentorial subarachnoidal Additionally, ASPECTS ratings on FDCT (figure $2 \mathrm{C}$ ) showed a mean difference of 0.5 points $(95 \% \mathrm{Cl} 0.12$ to 0.88$)$ space, and gray- white in the Bland-Altman plot (see online supplementary figure S1A) compared with ratings of MDCT images. When differentiation of the basal asked about their opinion on ischemic lesion detectability, raters stated that in $98 \%$ and $99 \%$ of cases

Table 1 Rating of cerebral structures

\begin{tabular}{|c|c|c|c|c|c|c|}
\hline Variable & & $\begin{array}{l}\text { Diagnostic } \\
\mathrm{n}(\%)\end{array}$ & $\begin{array}{l}\text { Identifiable but not diagnostic } \\
\mathrm{n}(\%)\end{array}$ & $\begin{array}{l}\text { Not identifiable } \\
\mathrm{n}(\%)\end{array}$ & $\begin{array}{l}\text { Total } \\
\mathrm{n}(\%)\end{array}$ & $\begin{array}{r}\text { Wilcoxon } \\
p \text { Value }\end{array}$ \\
\hline \multirow[t]{2}{*}{ Supratentorial ventricular system } & FDCT & $102(100)$ & $0(0)$ & $0(0.0)$ & $102(100)$ & - \\
\hline & MDCT & $102(100)$ & $0(0)$ & $0(0.0)$ & $102(100)$ & \\
\hline \multirow[t]{2}{*}{ Infratentorial ventricular system } & FDCT & $92(90)$ & $9(9)$ & $1(1)$ & $102(100)$ & 0.106 \\
\hline & MDCT & $98(96)$ & $3(3)$ & $1(1)$ & $102(100)$ & \\
\hline \multirow[t]{2}{*}{ Supratentorial subarachnoidal space } & FDCT & $98(96)$ & $4(4)$ & $0(0)$ & $102(100)$ & 0.813 \\
\hline & MDCT & $99(97)$ & $3(3)$ & $0(0)$ & $102(100)$ & \\
\hline \multirow[t]{2}{*}{ Infratentorial subarachnoidal space } & FDCT & $75(74)$ & $25(25)$ & $2(2)$ & $102(100)$ & 0.177 \\
\hline & MDCT & $85(83)$ & $14(14)$ & $3(3)$ & $102(100)$ & \\
\hline Gray-white differentiation of basal & FDCT & $97(95)$ & $5(5)$ & $0(0)$ & $102(100)$ & 0.563 \\
\hline ganglia & MDCT & $99(97)$ & $3(3)$ & $0(0)$ & $102(100)$ & \\
\hline \multicolumn{7}{|c|}{ Gray-white differentiation of insular FDCT 94 (92) 8 (8) 0 (0) 102 (100) 0.625 cortex MDCT 96 (94) 6 (6) 0 (0) 102 (100) } \\
\hline \multicolumn{7}{|c|}{ Gray-white differentiation of central FDCT 98 (96) 4 (4) 0 (0) 102 (100) 0.813 cortex MDCT 99 (97) 3 (3) 0 (0) 102 (100) } \\
\hline Gray-white differentiation of & FDCT & $57(56)$ & $37(36)$ & $8(8)$ & $102(100)$ & $<0.001$ \\
\hline cerebellum & MDCT & $88(86)$ & $13(13)$ & $1(1)$ & $102(100)$ & \\
\hline
\end{tabular}




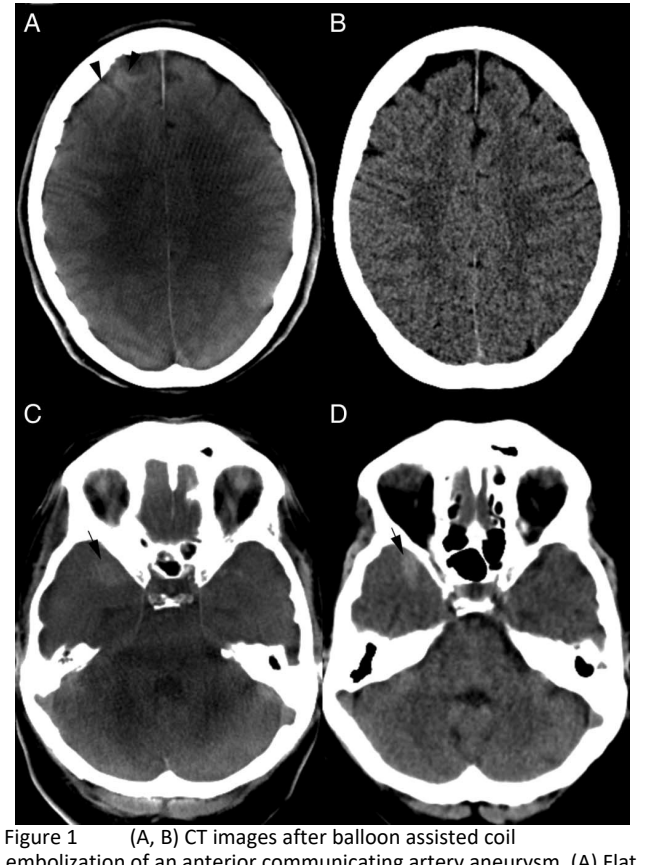

embolization of an anterior communicating artery aneurysm. (A) Flat detector CT (FDCT) shows a cortical hyperattenuation of the right frontal lobe (black arrowhead). A subarachnoidal hemorrhage (SAH) can be excluded on both FDCT (A) and follow-up multidetector C (MDCT) (B) images. Gray-white matter differentiation as well as exclusion of postinterventional ischemic lesions is feasible in both examinations. (C, D) Right temporal SAH. Blood is delineated on both FDCT (C) and MDCT (D) examinations (black arrows). Gray-white matter differentiation of the cerebellum is limited on FDCT (C) but the fourth ventricle is clearly depicted and an intraventricular hemorrhage can be excluded.

they were sure about the detection/exclusion of extended (ASPECTS $<5)$ ischemic lesions on FDCT and MDCT, respectively $(p=0.019)$.

SAH was diagnosed in 39 cases with FDCT; 37 were true positives, resulting in a sensitivity of $95 \%$ and a specificity of $97 \%$ (see online supplementary table S1; $\mathrm{p}<0.001$; AUC $0.96,95 \% \mathrm{Cl} 0.89$ to 0.98 ) (figure 3). One false negative FDCT scan included an SAH seen on a few slices (group with 2-12) on MDCT and one seen on several MDCT slices (group with 12-33). Thirty-three of the $34 \mathrm{IVH}$ cases were detected on FDCT ( $p<0.001$; AUC $0.98 ; 95 \% \mathrm{Cl} 0.93$ to 0.99 ). The one false negative FDCT scan included an IVH seen on a few slices (group with 2-4) on MDCT. All cases diagnosed with PH on MDCTwere also

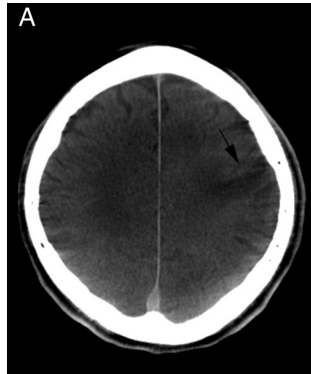

B

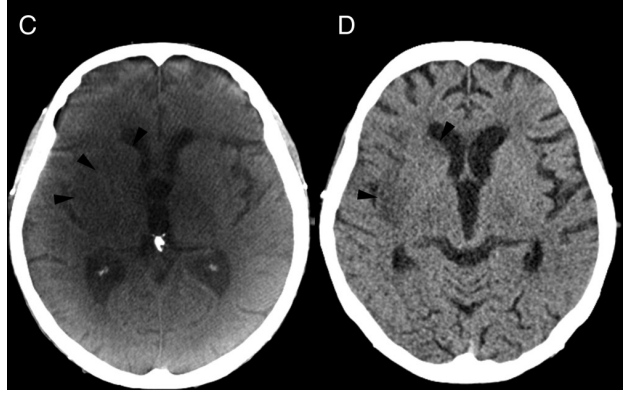

Figure 2

depicted and scored similarly on FDCT, resulting in $100 \%$ sensitivity and $99 \%$ specificity of FDCT for the detection of PH $(p<0.001$; AUC $0.99 ; 95 \% \mathrm{Cl} 0.95$ to 1.00 ). The median slice count of PH on MDCT was 10 (IQR 8-13). The mean difference between PH measurements on FDCT and MDCT was $-35.13 \mathrm{~mm}^{2}$ ( $95 \% \mathrm{Cl}-88.67$ to $18.39 \mathrm{~mm}^{2}$, see online supplementary figure $\mathrm{S} 1 \mathrm{~B})$. In the supratentorial region, raters found 'very good' image quality for hemorrhage detection in $87 \%$ of FDCT scans compared with $93 \%$ of MDCT detector CT (FDCT) scan after carotid artery stenting (A, black arrow). No acute ischemic lesions were detected on this scan. The same lesion can be confirmed on multidetector $\mathrm{CT}$ (MDCT) (B, black arrow). (C, D) CT images prior to thrombectomy and at follow-up. Acute ischemic lesions can be seen on non-contrast FDCT (C, black arrowheads) performed prior to thrombectomy. An intracranial hemorrhage can be excluded and an Alberta Stroke Program early CT Scale (ASPECTS) score of 7 can be rated on FDCT images. Ischemic lesions are confirmed on follow-up MDCT images (D, black arrowheads) after rapid reperfusion.

examinations $(p=0.266)$. A statistically significant difference was shown in the same category of scores in the infratentorial region, with $58 \%$ of 'very good' scores on FDCT compared with $78 \%$ on MDCT $(\mathrm{p}=0.002)$. When asked about their subjective judgement on
(A) An

older

small cortical
infarction is depicted on the flat 
hemorrhagic lesion detectability, raters stated that in $98 \%$ and $99 \%$ of $\quad 0.81$ ) while lower values were documented between the experienced ( lesions on FDCT and MDCT, respectively $(p=1)$.

physician and the medical student $(\mathrm{r}=0.71 ; 95 \% \mathrm{Cl} 0.69$ to 0.73$)$ as well

Metal artifacts were delineated in $22 \%$ of FDCT scans, with only one case with significant degradation of image quality. Motion, ring, and burn-in artifacts were visible on $26 \%, 23 \%$, and $24 \%$ of FDCT scans, respectively. Streak artifacts were present on the majority of FDCT scans $(72 \%)$. However, most artifacts only slightly affected the image quality of FDCT scans.

as the inexperienced physician and the medical student $(r=0.69 ; 95 \%$

The highest correlation coefficient between overall raters' scores

\section{DISCUSSION}

The primary use of FDCT to date has been limited to detection of periinterventional complications, such as SAH after coiling

was documented between the two physicians $(r=0.80 ; 95 \% \mathrm{Cl} 0.79$ to 


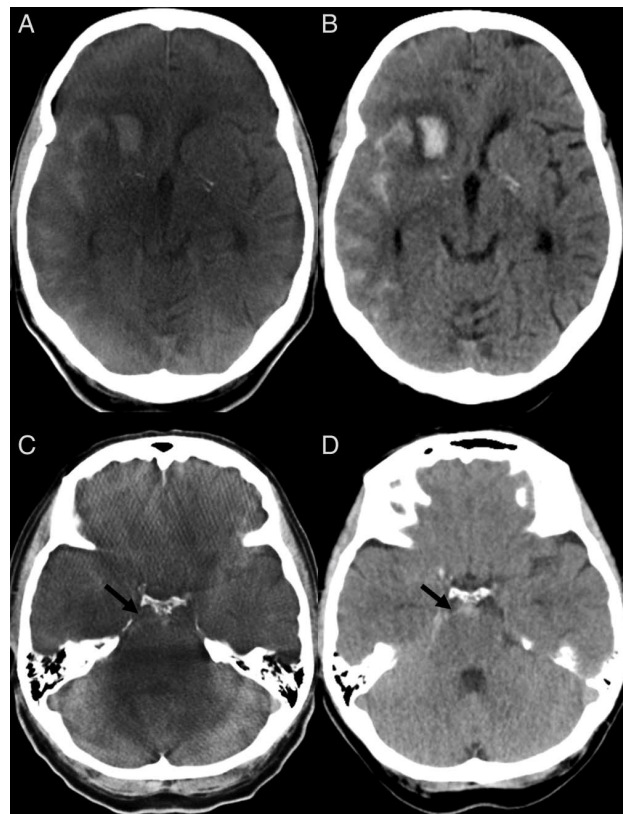

Figure 3 A parenchymal hemorrhage and subarachnoidal hemorrhage (SAH) can be diagnosed on flat detector CT (FDCT) (A) and verified on follow-up multidetector CT (MDCT) (B). (C, D) A small and verified on follow-up multidetector CT (MDCT) (B). (C, D) A small images. The same findings were delineated on follow-up MDCT (D, black arrow).

of an intracranial aneurysm or $\mathrm{PH}$ after thrombectomy of a large vessel occlusion. Multiple studies have documented the limitations of previous generations of FDCT in the detection of intracranial sized patients. In addition, it provides a full set of quadratic and generally smaller focal spot sizes, thus enhancing image sharpness in all viewing directions. As in previous studies, delineation of supratentorial ventricles was excellent with FDCT. Compared with past generations of FDCT, a significant improvement was observed in the infratentorial region, as we found $74 \%$ and $90 \%$ of FDCT scans to be of 'diagnostic' quality regarding depiction of subarachnoidal spaces and of the fourth ventricle, respectively ( $29 \%$ and $61 \%$ in a previous study). ${ }^{4}$ However, with limited diagnostic quality in $26 \%$ of FDCTscans, detection of small infratentorial SAH can be challenging. This fact should be taken into consideration in future studies evaluating the use of FDCT as a primary triage tool in stroke. The radiation dose of the FDCT scan used in our study is comparable with incremental MDCT examinations with an effective dose of approximately $2.5 \mathrm{mSv} .{ }^{89}$

The primary limitation of our study is its retrospective character. As FDCTand MDCT scans were acquired with a time delay, we cannot exclude false negative results due to new hemorrhage or demarcation of ischemic lesions. Two false positive SAH cases on FDCT were a result of contrast media extravasation after a long intervention which was falsely rated as an SAH on FDCT but resolved after a few hours on MDCT. Lastly, FDCT detectability of ischemic lesions and gray-white differentiation may have been enhanced by the presence of contrast media, in cases where FDCTwas performed postinterventionally.

Our results may impact the management of acute stroke patients. After publication of the 'unhappy triad' of negative trials in 2013, time from hospital admission to reperfusion was identified as a reason for the negative results, in addition to the use of older devices and low reperfusion rates. ${ }^{10-12}$ The authors of the 'unhappy triad' trials reported significant delays to initiation of endovascular treatment, with up to $120 \mathrm{~min}$ from imaging to groin puncture. Inhospital times were much improved in the five positive randomized trials published in 2015, with a median door to groin time of 104 min. However, only $13 \%$ and $4 \%$ of the randomized patients were treated within the 'ideal' SNIS 2015 intervals of $\leq 60 \mathrm{~min}$ for door to groin time and $\leq 30$ min for imaging to groin time. ${ }^{13}$ Even with a streamlined process, Frei et $\mathrm{al}^{14}$ recently reported a median door to groin time of $71 \mathrm{~min}$ for direct admission patients undergoing triage with MDCT.

Implementation of a one stop management for stroke patients could lead to a reduction in inhospital times. In our department, we hemorrhages and ischemic lesions. ${ }^{2-4}$ Such limitations have restricted the use of FDCT as a primary imaging modality in acute stroke imaging because administration of intravenous recombinant tissue plasminogen activator as the gold standard stroke therapy up to 2015 required reliable exclusion of hemorrhage prior to therapy initiation. In the new era of acute stroke treatment, reliable differentiation between ischemic and hemorrhagic stroke remains a fundamental element of multimodal stroke imaging. Depiction of early ischemic signs has lost importance within the first 6 hours after symptom onset, as even patients with extended ischemic lesions (ASPECTS 0-5) may profit from

thrombectomy. ${ }^{56}$

We found FDCT to be as reliable and accurate as MDCT in the detection of intracranial hemorrhage. High sensitivity and specificity was shown for SAH and IVH. Two of the false negative cases were associated with extensive motion artifacts on FDCT and were visible on only a few MDCT slices. All PH, regardless of size or intracranial location, were depicted and diagnosed on FDCT. Gray-white differentiation in the supratentorial region was feasible with the latest generation of FDCT, as there was no statistically significant difference in scores compared with MDCT. Moreover, detection of ischemic lesions was much improved compared with previous generations of FDCT, as we found $71 \%$ sensitivity compared with the gold standard MDCT. ${ }^{4}$

The new generation of FDCT promises much better gray- white differentiation due to the high dynamic range flat detector, enabling four times more gray value differentiation, approaching the contrast resolution of conventional MDCT. Artifacts near the skull base are reduced due to new reconstruction algorithms. ${ }^{7}$ Furthermore, the latest generation of $\mathrm{X}$-ray tubes enables higher maximum tube current than earlier systems, which reduces voltage variation and enables better penetration during the acquisition, especially in larger estimated a time gain of approximately $30 \mathrm{~min}$ if we bypass MDCT and transport patients with suspected large vessel occlusions directly to the angio suite (Psychogios MN, unpublished data, 51th Meeting of the German Society of Neuroradiology, http://dgnr.conference2web.com/content/748/details?from view=all, 2016). The addition of flat detector CT perfusion or multiphase flat detector CT angiography allows the acquisition of multimodal CT within the angio suite as a complete stroke imaging battery without relevant limitations compared with MDCT multimodal imaging. ${ }^{15} 16$

\section{CONCLUSIONS}

The latest generation of FDCT is a reliable and accurate tool for the detection of intracranial hemorrhage. Gray-white differentiation is feasible in the supratentorial region but seems limited for the infratentorial brain.

Contributors Guarantor of the integrity of the entire study: M-NP. Study concepts: MK and M-NP. Study design: M-NP, JRL, IT, and ACH. Definition of intellectual content: M-NP, IT, and JRL. Literature research: M-NP, JRL, $\mathrm{DB}$, and KS. Clinical studies: IT, JRL, DB, KS, and ACH. Data acquisition: IT, JRL, DB, and KS. Data analysis: M-NP, MK, IP, and ACH. Statistical analysis: M-NP, JRL, and IP. Manuscript preparation: M-NP, JL, and JRL. Manuscript editing: M-NP and DB. Manuscript review: M-NP, MK, JL, and IP.

Competing interests The Department of Neuroradiology, University Medicine Goettingen, has a research agreement with Siemens Healthcare $\mathrm{GmbH}$, Forchheim, Germany.

Ethics approval The study was approved by theethics committee of University Medicine Goettingen. The ethics committee of our hospital waived the need for a formal application or informed patient consent due to the retrospective design of this study.

Provenance and peer review Not commissioned; externally peer reviewed.

Previous or future presentations 
Danksagung

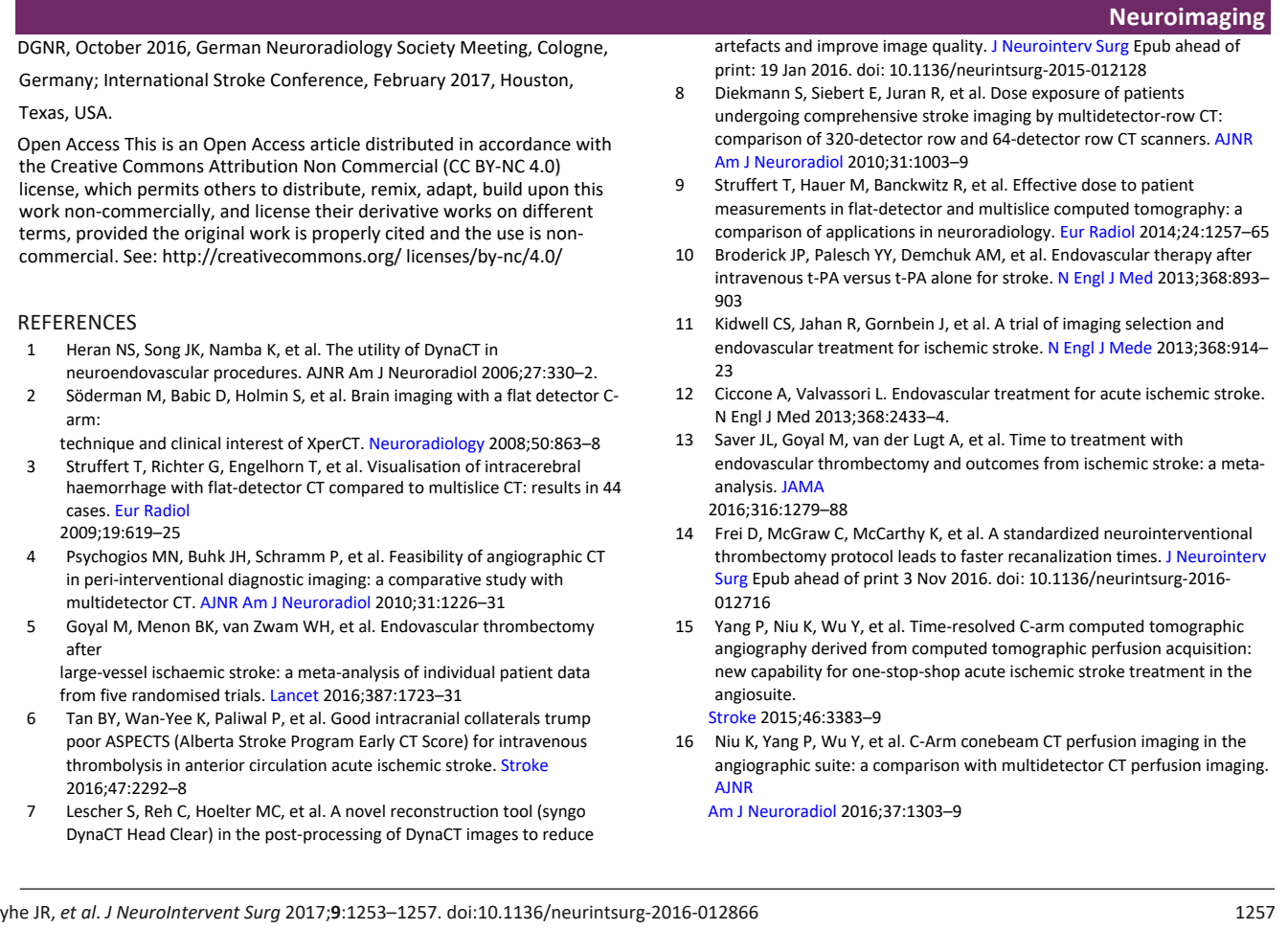

Texas, USA.

Open Access This is an Open Access article distributed in accordance with worms, provided the or $/$ ina work is propery cited and the use is non-

FERENCES

Psychogios MN, Buhk JH, Schramm P, et al. Feasibility of angiographic Cr s. Lancet 2016;387:1723-3 2016:47:2292-8

Lescher S, Reh C, Hoelter MC, et al. A novel reconstruction tool (syngo 257

\section{Danksagung}

Ich möchte mich insbesondere bei meinem Doktorvater Prof. Dr. Marios Psychogios für die hervorragende Unterstützung und Förderung während des gesamten Prozesses dieser Arbeit bedanken.

Mein Dank gilt darüber hinaus Dr. Ioannis Tsogkas und Amélie Hesse, die mich durch ihre Auswertung ebenfalls sehr unterstützt haben. 
Lebenslauf

\section{Lebenslauf}

Ich wurde am 25.08.1992 als Tochter von Henning und Susanne Leyhe in Göttingen geboren. Nach meiner schulischen Ausbildung an der Herkulesschule Kassel und am Friedrichsgymnasium Kassel begann ich im Oktober 2012 mein Medizinstudium an der GeorgAugust- Universität in Göttingen, welches ich im Juni 2019 erfolgreich abschließen konnte.

Als Mitglied der „Stroke Research Group Göttingen“ konnte ich seit 2015 an verschiedenen Projekten unter anderem an dem Thema dieser Dissertation mitarbeiten und erste wissenschaftliche Erfahrungen sammeln. Darüber hinaus habe ich den studentischen Rufbereitschaftsdienst der Interventionellen Neuroradiologie mitbegründet und von 2016 bis 2018 geleitet.

Ebenfalls habe ich mich im Bereich der akademischen Selbstverwaltung und der Fachschaft Medizin und der Hochschulgruppe Unabhängige Mediziner Göttingen e.V. engagiert und unter anderem die Studierenden im Fakultätsrat und der Studienkommission vertreten.

Mein Praktisches Jahr habe ich im Zentralkrankenhaus in Bozen, Italien, am Mater Misericordiae University Hospital in Dublin, Irland, am St. Bernward Krankenhaus in Hildesheim und an den DRK Kliniken Nordhessen Kassel absolviert. Seit September 2019 arbeite ich in der Klinik für Neurologie und klinische Neurophysiologie am St. Bernward Krankenhaus in Hildesheim als Assistenzärztin. 